\title{
Model-Driven and Pattern-Based Integration of Process-Driven SOA Models
}

\author{
Uwe Zdun and Schahram Dustdar \\ Distributed Systems Group \\ Information Systems Institute \\ Austria \\ $\{$ zdun $\mid$ dustdar\}@infosys.tuwien.ac.at
}

\begin{abstract}
Service-oriented architectures (SOA) are increasingly used in the context of business processes. However, the modeling approaches for process-driven SOAs do not yet sufficiently integrate the various kinds of models relevant for a process-driven SOA - ranging from process models to software architectural models to software design models. We propose to integrate process-driven SOA models via a model-driven software development approach that is based on proven practices documented as software patterns. We introduce pattern primitives as an intermediate abstraction to formally model the participants in the solutions that patterns convey. To enable model-driven development, we develop domain-specific modeling languages for each kind of process-driven SOA model - based on formal metamodels that are extended with the pattern primitives. The various process-driven SOA models are integrated in a model-driven tool chain via the meta-models. Our tool chain validates the process-driven SOA models with regard to the constraints given by the meta-models and primitives.
\end{abstract}

\section{Introduction}

Many service-oriented architectures (SOA) provide a Service Composition Layer that introduces a process engine (or workflow engine) as the top-level layer [35]. Services realize individual activities in the process (aka process steps, tasks in the process). This kind of architecture is called process-driven SOA. The main goal of process-driven SOAs is to increase the productivity, efficiency, and flexibility of an organization. This is achieved by aligning the high-level business processes with the technical IT services. That is, the business goals get closer integrated with the IT architecture. Organizational flexibility can be achieved because explicit business process models are easier to change and evolve than for instance business processes that are hard-coded in the program code. In the long run the goal is to enable business process improvement through IT.

Dagstuhl Seminar Proceedings 06291

The Role of Business Processes in Service Oriented Architectures

http://drops.dagstuhl.de/opus/volltexte/2006/820 
One of the most important characteristics of SOAs suggests heterogeneity of technologies and integration across vendor-specific technologies [29]. This, however, yields an important challenge for modeling process-driven SOAs: Many modeling domains need to be considered and the different kinds of models need to be integrated. For instance, among many other modeling domains, we need to consider component architectures, message flows, transactions, security, workflows/business processes, programming language (snippets), business object designs, and organizational models. In addition, application domains introduce domain models, such as a banking or insurance domain models. Furthermore, implicit or explicit models for integrating existing legacy systems are needed: They often introduce an additional modeling domain because they are built on different concepts than the rest of the SOA.

In other words, a central challenge for modeling process-driven SOAs is that we generally need to integrate different kinds of models and abstractions. This problem is challenging because so far there is no formal and precise modeling approach for integrating all these kinds of models.

In addition to the missing integration of process-driven SOAs models for different modeling domains, even within one and the same domain integration is needed. For instance, in the domain of workflow or business process languages many different languages and tools exist, with highly different characteristics. If, for example, a company works on projects for different customers or two departments/companies need to integrate their IT (e.g., because of a fusion), it is not unlikely that different workflow or business process modeling languages and tools are used. Similar situations can occur in all the other modeling domains as well.

Finally, the executable languages used to implement the models (e.g., process execution languages like BPEL or programming languages) are also diverse. In similar situations, as the ones where modeling language integration is needed, also an integration of the executable languages - both within and across modeling domains - is needed.

In this paper, we propose a concept for a model-driven tool chain that addresses these challenges through modeldriven software development (MDSD) [26, 12]. Our concept is based on the formal specification of the models in domain-specific languages (DSL) which are formally defined in terms of meta-models. The code in the executable languages is generated from the models expressed in the DSLs. That is, the integration issues raised above are solved at the meta-model level.

Our tool chain and MDSD concepts break the integration issues down to the problem of finding adequate metamodels for representing all concerns to be modeled in the various modeling languages used in the modeling domains. In this paper, we propose to develop the meta-models according to proven practices that can be found in existing process-driven SOAs. Our assumption is that using proven practices as a foundation for meta-modeling leads to a close match between the modeling abstractions and the existing modeling languages (and thus the real world requirements in the field).

In our approach, the software patterns concept is used to describe the proven practices. Software patterns capture reusable design knowledge and expertize that provides proven solutions to recurring software design problems that arise in particular contexts and domains [23]. A software pattern, however, is typically described in an informal form and cannot easily be described formally, e.g., by using a parameterizable, template-style description. Hence, as such, 
patterns are not usable in formal meta-models. We remedy this problem by introducing an intermediate abstraction, called pattern primitives. A pattern primitive is a fundamental, formalizable modeling element in representing a pattern.

Our general approach to apply pattern primitives for process-driven SOAs is to use one kind of formal modeling language for all kinds of flow models that are used in a process-driven SOA - and extend it with additional modeling concepts where necessary to connect to other kinds of models. The connection between the various kinds of models and the validation of the models (with regard to model integrability and consistency in and across the modeling domains) is the main task of our model-driven tool chain concepts. To demonstrate our approach, we will use a formalizable subset of UML2 and OCL to develop one formal modeling language to depict the various refinements of processes in process-driven SOA models, even though our approach in general is not depending on the use of the UML.

In this paper, we first provide the background on MDSD and patterns/pattern primitives in Section 2 to lay out the foundations of our approach. Next, in Section 3 we explain the concepts and architecture of our model-driven tool chain to give an overview of our approach. In Section 4 we explain how we use meta-models to integrate processdriven SOA models across modeling domains. Then in Section 5 we explain the pattern primitives approach for process-driven integration of services using flow abstractions as the primary modeling domain used in our approach for modeling process-driven SOAs. In Section 6 we demonstrate how architectural abstractions - as one example of another modeling domain - can be integrated with the flow abstraction models. We explain all primitive models with running examples from a pattern language for process-driven integration of services, which we have implemented in our MDSD tool chain to validate our approach. Finally, we discuss related work, evaluate our approach in comparison to related work, and conclude.

\section{Background}

Before presenting our concepts for model-driven and pattern-based integration of process-driven SOA models, we want to briefly explain the model-driven software development and software patterns concepts that we use as the foundations for our approach.

\subsection{Model-driven Software Development}

Our approach to model-driven software development (MDSD) [26, 12] for process-driven SOAs is based on the notion of domain-specific languages (DSL) for modeling the various types of models. Domain-specific languages are "small" languages that are tailored to be particularly expressive in a certain problem domain. The DSL describes knowledge via a graphical or textual syntax (referred to as the DSLs concrete syntax [12]), which is tied to domainspecific modeling elements through a formal language meta-model (referred to as the DSLs abstract syntax [12]). That is, the DSL elements are defined in terms of a meta-model that can be instantiated in concrete application 
models. The application models are defined in the DSL's concrete syntax, which represents the abstract syntax defined in the meta-model.

In our approach, we use or introduce meta-models that are representing a modeling domain. The meta-models presented in this paper are based on the UML2 meta-model (and extensions of it): For example we use UML2 activity diagrams to model flow abstractions and UML2 class/component diagrams to model the object-oriented design and architecture models. But any other meta-model can be used in a similar way. Examples for concrete syntaxes are a graphical UML model or an XML file specifying a process model.

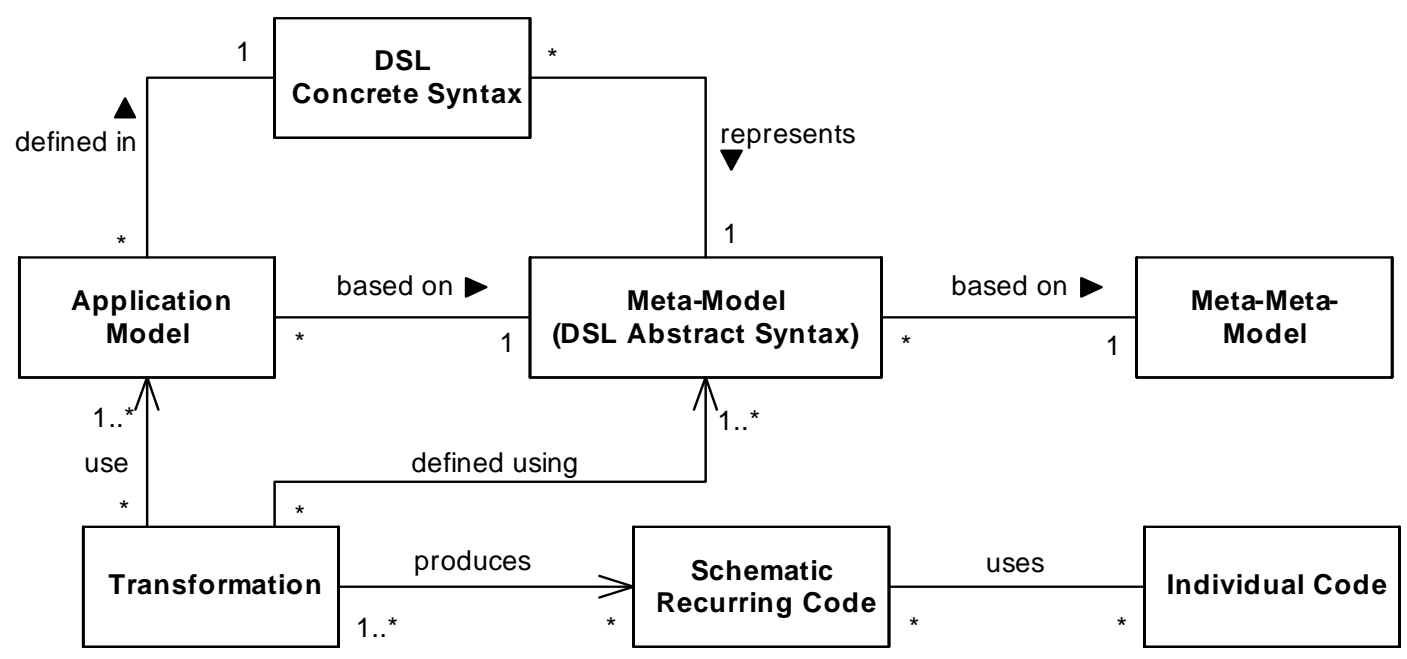

Figure 1. Relations of Artifacts in MDSD

Meta-models are defined in terms of a meta-meta-model. In UML, for instance, this is MOF. Most MDSD tools support their own meta-meta-model, which basically represents a mapping from meta-model definitions to the implementation of the MDSD tool chain. Below, in the examples from our prototype, we use a simple meta-meta-model to define both the UML2 meta-model and pattern primitives extensions. It is not particularly important for our approach, which meta-meta-model is used, there just must be some way to express the relationships between a meta-model and the implementation code. The meta-meta-model is not visible to developers who build application models, but only to those who build meta-models.

Each MDSD tool introduces some way to specify transformations. There are different kinds of transformations, such as model-to-model transformations or model-to-code transformations. There are also different ways to specify transformation, such as transformation rules, imperative transformations, or template-based transformations. In any case, the ultimate goal of all transformations in MDSD tools is to generate code in executable languages, such as programming languages or process execution languages. The MDSD tools are used to generate all those parts of the executable code which are schematic and recurring, and hence can be automated. Of course, some code must be hand-written either because it is individual code for a system or the semantics of the code are not fully covered by the DSLs (yet). The individual code and the generated code use each other and interact through well defined interfaces.

Figure 1 summarizes the relations of artifacts in MDSD. This short introduction to the terms used in MDSD should 
suffice for this paper. We will provide examples for the various concepts in the text below, as we use them. Please refer to $[26,12]$ for a thorough introduction to the MDSD approach.

\subsection{Software Patterns and Pattern Primitives}

Software patterns and pattern languages have gained wide acceptance in the field of software development, because they provide systematic reuse strategies for design knowledge [23]. Each pattern is a three-part rule, which expresses a relation between a certain context, a problem, and a solution [3]. A pattern language is a collection of patterns that solve the prevalent problems in a particular domain and context, and, as a language of patterns, it specifically focuses on the pattern relationships in this domain and context.

Patterns informally describe many possible variants of one software solution that a human developer or designer can recognize as one and the same solution. A pattern encodes proven practices for particular, recurring design decisions. A pattern language can thus be seen as a language of design decisions that one can follow in a number of possible sequences. Hence, patterns encode recurring design decisions, give them a name that can be used throughout a development team, allow development team members to trace the design considerations in the design decisions by following the sequences in the pattern language, etc.

Even though these properties of the pattern approach are highly valuable in the software design process, they also make pattern instances hard to trace in the models and implementations. To overcome this problem, we introduced an approach to document formalizable primitive abstractions that can be found in the patterns [34]. Documenting pattern primitives means to find precisely describable modeling elements that are primitive in the sense that they represent basic units of abstraction in the domain of the pattern. Our original pattern primitives concept presented in [34] is only targeted at modeling architectural patterns. That is, in the architectural realm, basic architectural abstractions like components, connectors, ports, and interfaces are used. An interesting challenge in describing the pattern primitives for the patterns of process-driven SOA is that this area is characterized by the fact that we need to understand various design and architecture concepts, as well as various design and implementation languages, in order to be able to model a process-driven SOA design fully. Also, other aspects like organizational roles must be considered. This is an important difference to the area of general architectural patterns.

In this paper, we model pattern primitives using UML 2.0 extensions because the UML has become the "lingua franca" of software design and is vastly supported by tools. We specify an extension of a UML 2.0 metaclass for each elicited primitive, using the standard UML extension mechanisms: stereotypes, tag definitions, and constraints. We use the Object Constraint Language (OCL) to formalize the constraints and provide precise semantics to the primitives.

\section{Model-driven Tool Chain: Concepts and Architecture}

In our concept, similar artifacts must be produced for each modeling domain. As many modeling domains need to be considered to adequately model a process-driven SOA, we will first describe the general activities which must 
be performed for each individual modeling domain - before a model-driven development of process-driven SOAs in this modeling domain is possible. Next, we will give an overview of our integration concepts for integrating models across modeling domains.

\subsection{Model-driven Design Process}

A model-driven design process, according to our approach, should loosely follow the activities described below. Please note that our approach does not require any particular order of these activities.

1. Elicitation of input languages/models: In each modeling domain, there might be multiple input languages or models which are needed for the process-driven SOA. For instance, if different UML tools are used, different XMI exports of these tools must be considered for UML models. Another example is the area of business process and workflow modeling languages, where a plethora of tools exists which use different standard and non-standard process and workflow modeling languages. Even the same standard language might be interpreted differently by different tool vendors. Similar situations exist in other modeling domains. It is hence important to elicit the relevant input languages/models, and maybe identify a sub-set of them to be reflected in the DSLs. Please note that sometimes no external tool should be used for modeling, but an inhouse development or an external tool can be extended with export formats. Then it is possible to model directly in the DSL (i.e., the DSL concrete syntax is the only input format for the modeling domain).

2. Elicitation of output languages (aka execution languages): Sometimes only one target execution language should be used as an output for a particular modeling domain. But in other cases, the same models should be used for generating code in different execution languages. For instance, if a company develops code for different process engines (e.g., in different projects), different business process languages might be used as execution languages by these engines or different dialects of one language. A similar situation can also occur, if different programming languages or platforms are to be supported.

3. Development or definition of an MDSD tool chain: There is a common workflow for model-driven code generation following our approach: the input languages/models need to be read and transformed into DSLs which are defined using meta-models. Then the DSL code should get validated according to the meta-models and constraints defined on them. Finally, code in the target output languages should get generated. In between many other steps are possible, such as model transformations. The generation tool chain must support a way to define meta-models. It must also provide a constraint language to define structural constraints at the meta-level (i.e., on meta-models). The tool chain needs to be extensible with input and output languages/models, e.g., via plugins. It must provide a means to flexibly assemble the generation workflow and the plugins. There are many existing code generators that provide these features (an open source example is openArchitectureWare [22]), but of course it is also possible to custom-build parts of the generation tool chain to realize the concepts. In our prototype we use the language Frag $[33,32]$ for the definition of DSLs and meta-models, because it is 
especially designed for this task. The language is also used for the implementation of the constraint language and the model validator.

4. Definition of meta-models (abstract syntax of modeling DSL): For each DSL we need to define a meta-model which describes the abstract syntax of the DSL. That is, the meta-model defines the entities (domain concepts) that are represented by the language, their relationships, and constraints on them. In this paper, we use extensions of the UML2 meta-model because it pre-defines many of the elements that we require in the model types. We extend it with pattern primitives via UML profiles. OCL is used to define constraints. However, any other kind of meta-models can be used as well.

5. Definition of a concrete syntax for the DSL: The concrete syntax defines how the DSL meta-model is mapped to language elements and a grammar. The concrete syntax can either be textual or graphical. In this paper, we use the Frag textual syntax as one common syntax because its easy to parse and to map onto Frag meta-models. Though it is quite useful to use one common syntactic base for all concrete syntaxes of the DSLs in order to reduce the learning effort for developers, this is no prerequisite of our approach. Any suitable concrete syntax can be chosen.

6. Development of transformation plugins for each input language: If the input languages differ from the DSL, a mapping between input language and DSL must be defined. Also a plugin for the generator to transform the input language into the concrete syntax must be defined.

7. Development of transformation plugins for each output language (aka execution language): Execution language code is generated from the models written in the DSLs. For each combination of DSL and execution language, a plugin for the generator should be developed, so that the generator can automatically generate code in the execution language that conforms to the model.

As an example, let us consider our MDSD tool chain for process-driven integration of services. Please note that many other configurations are possible. For instance, other input and output models or DSL syntaxes can be used in the same way.

Our tool chain is depicted in Figure 2. We mainly use UML2 models that are extended with UML2 profiles for modeling the pattern primitives as inputs. These UML2 models can either be developed with UML tools (with XMI export) or directly in the textual DSL syntax. If a UML tool is used, the XMI export is transformed into the textual DSL syntax.

We use Frag $[33,32]$ as the syntactic foundation of the textual DSLs and for defining the meta-models of the DSLs. Frag's main goal is to provide a tailorable language. Among other things, Frag supports the tailoring of its object system and the extension with new language elements. Hence, Frag provides a good basis for defining a UML2-based textual DSL because it is easy to define a meta-meta model on top of which we can define the UML 
meta-classes. Frag automatically provides us with a syntax for defining application models using the UML2 metaclasses. In addition to UML2 meta-models and the meta-meta-model, we have defined a constraint language which follows the OCL's constructs.

The model validator gets all input models and validates the conformance of the application models to the metamodels. It also checks all OCL constraints. Especially, that means it checks the constraints given by the pattern primitive definitions.

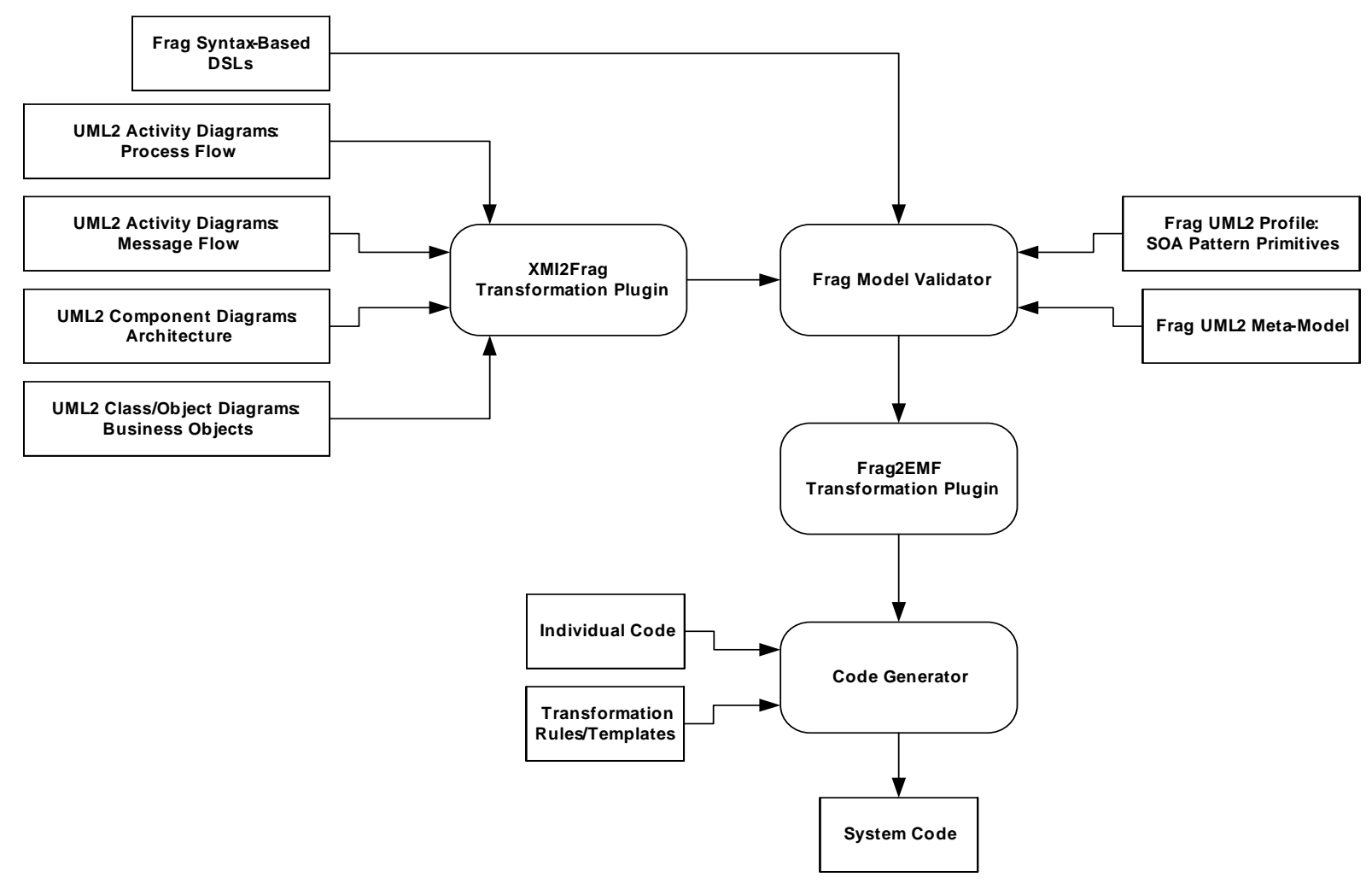

Figure 2. Tool Chain Overview

After the model is validated it is transformed into an EMF model, which is understood by the code generator. We then generate code in executable languages, such as Java and BPEL, using the code generator.

\subsection{Model Integration Concepts: Meta-meta-model Based Integration}

The model-driven design activities and architecture described in the previous section only concentrate on the individual modeling domains. For integration of the models, we propose further integration concepts that extend the general model-driven approach. Because they are independent of external tools, languages, or models, in our concept, the central point of integration are the meta-models that we need to define for the DSLs. Also, they are located at the central place of the model-driven architecture: at the point in the tool chain where all different models are assembled.

We propose to define the meta-models on top of one common meta-meta-model. The meta-meta-model can be 
very simple, or more elaborate like MOF. The most important criterion for the meta-meta-model is that the elements of the meta-meta-model allow the model validator to check models against the meta-models. In addition, it should be possible to define a constraint language using the meta-meta-model, with which models can be constrained at the meta-level and hence validated at the model level.

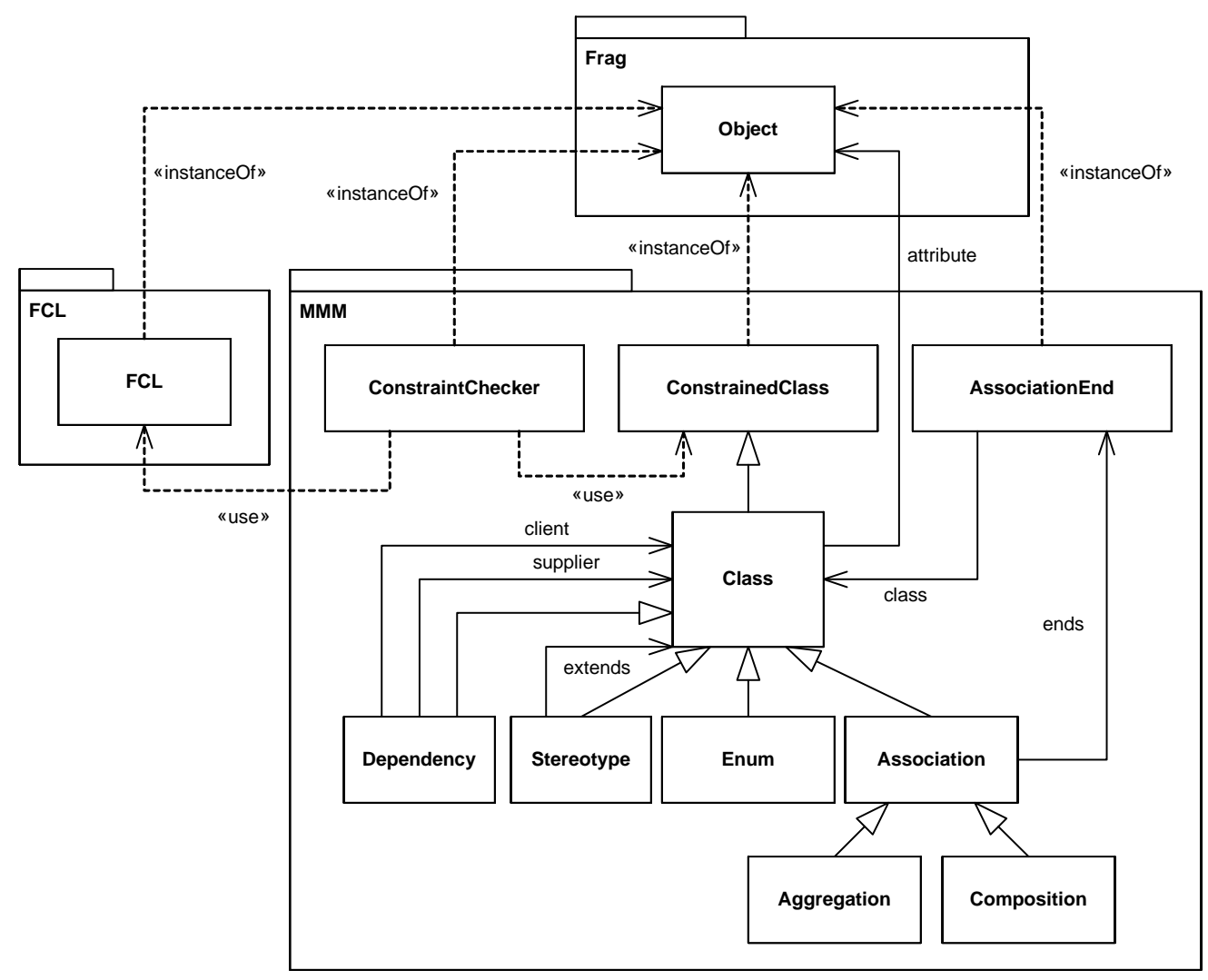

Figure 3. Meta-meta-model Excerpt

As an example, Figure 3 shows the relevant excerpt of the meta-meta-model that we use in Frag to define UML2 meta-models. This meta-meta-model is very simple and reuses Frag's language features wherever possible. It is derived from the most general class in the Frag object system: object. The meta-meta-model classes are subclasses of ConstrainedClass which allows to add OCL-style constraints to classes. The convenience class ConstraintChecker looks up all ConstrainedClass instances via reflection and checks the constraints. Constraints are specified in a language similar to OCL (defined using the class FCL). The meta-models are defined using Class. We introduce also a number of relationships between classes: Dependencies, Associations, Compositions, and Aggregations. In addition, typed attributes can be specified. Please note that we do not define the generalization relationship, because multiple inheritance is suitably predefined by Frag and we can reuse this implementation. The Stereotype class defines the UML2 extends-relationship; that is, it allows to extend meta-classes. Enum is a convenience class to define Enumeration types. 


\subsection{Model Integration Concepts: Proven Practices Based Integration}

Besides the common meta-meta-model concept, we use proven practices descriptions as the second central model integration concept: As explained above, we use software patterns to describe proven practices of process-driven SOAs. Patterns have two characteristics which make them useful for model integration across modeling domains:

- Patterns describe recurring solutions in a particular problem domain in an informal and holistic manner. Hence, in contrast to most formal modeling notations, they do not abstract from details that go beyond a specific modeling domain's abstractions, but instead explain the full solution. That is, if the solution has, for instance, implications for the workflow, the organization, and the software architecture, all these solution elements are described.

- As proven practice descriptions, patterns encode the recurring themes in the same kinds of models. Hence, they are also a good basis for defining a common meta-model for a modeling domain, because patterns typically describe the established, stable abstractions that are used across different modeling approaches and execution languages.

Because patterns are defined only informally, we use pattern primitives as an intermediary abstraction to represent the primitive concerns in the patterns formally. At this point, it is very important that we use a common meta-metamodel and a common constraint language to define the meta-models that represent the abstract syntaxes of the DSLs: This way, the primitives can be connected via constraints, and also primitives that cut across different models can be defined. The model validator can check all structural properties and constraints in the complete model, even if modeling domains are crossed.

\section{Meta-models for Process-Driven Integration of Services}

There are many modeling domains that play a role for a process-driven SOA. In our tool chain we have so far concentrated on a sub-set of these domains that deals with the integration of processes and services. In this domain, the following types of languages/models are typically used:

- the component architectures,

- the message flow specifications,

- the workflow or business process languages,

- programming languages and snippets written in programming languages,

- and business object design models. 
For our tool chain, we model both, message flow specifications and workflow or business process languages, using extension of UML2 activity diagrams ${ }^{1}$. Component architectures are modeled using UML2 component diagrams. Business object design models are modeled using UML2 class diagrams. In this paper, we will concentrate on examples that illustrate the integration of component architectures and flow abstractions, but the integration with business object design models can be done analogously. Programming language snippets are introduced as individual code (as explained in Section 3.1, cf. Figure 2).

As an example for a meta-model definition let us consider the central flow abstractions: The different models that are relevant for a process-driven SOA come together in various kinds of "flow" models. There are flow models for long-running business processes, activity steps in long-running processes, short-running technical processes, and activity steps in short-running technical processes. Even though these flow models have highly different semantic properties, they share the same basic flow abstraction concept, and at the same time they are a kind of glue for all the other models that are involved in a process-driven SOA (such as architecture and design models).

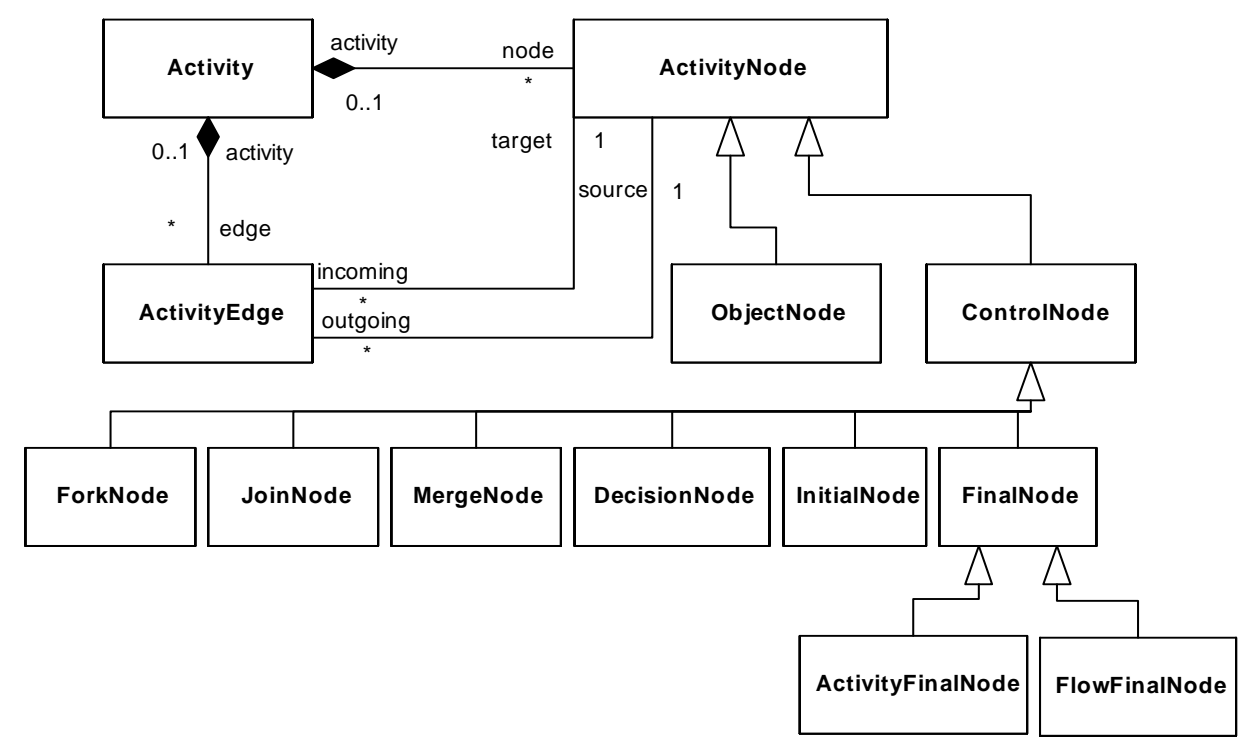

Figure 4. UML2 Activity Diagram Meta-model Excerpt

Figure 4 shows the excerpt of the UML2 activity diagram meta-model that is relevant for the following examples in this paper. We can define this meta-model by instantiating the meta-meta-model classes from Figure 3. In the Frag syntax, the same meta-model looks as follows:

MMM::Class create Activity

MMM::Class create ActivityNode

\footnotetext{
${ }^{1}$ Please note that in both cases, long running business processes and short running technical processes, the UML2 activity diagrams must be extended to depict relevant additional information. For instance, to represent long running business processes properly we must also depict organizational roles, organizational structures, business resources, etc. To represent short running technical processes properly, we must add technical details, such as protocol information, technical resources, or message queue details. In the examples of this paper, we omit these details because we want to concentrate on the process/service interaction aspects.
} 


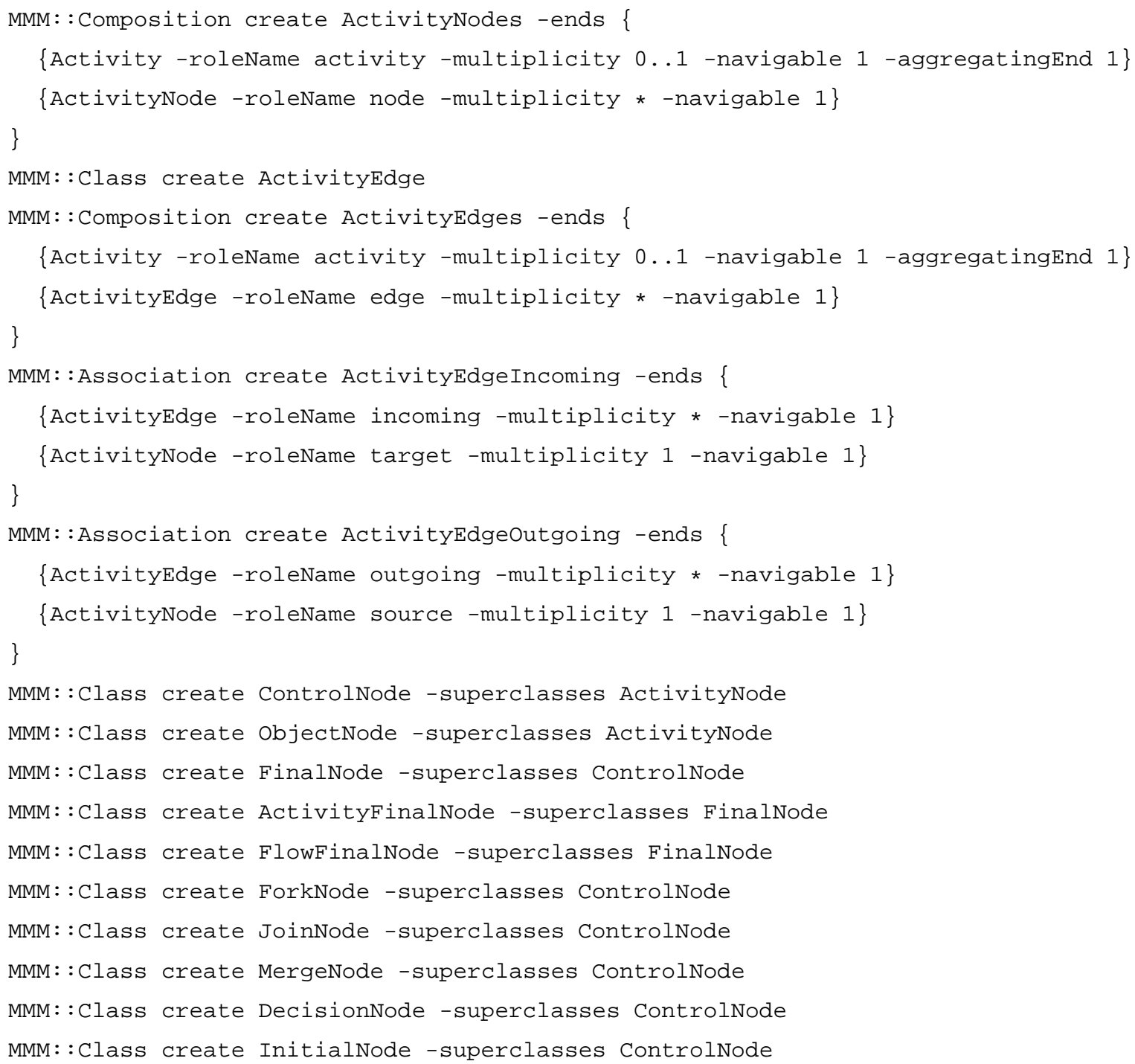

As the meta-models for class and component diagrams are defined pretty much in the same way, we omit them here.

\section{Patterns and Pattern Primitives for Process-Driven Integration of Services}

To realize our approach, we must next discuss how to extend the meta-models with pattern primitive extensions. Before we can go into detail, we first give an overview of the pattern language from which we derive the pattern primitives.

\subsection{Overview: Patterns for process-oriented integration of services}

In this section, we give an overview of the pattern language for process-oriented integration of services (for details please refer to [14]). In the next section, we present the primitives that we have mined from this pattern language. The pattern language basically addresses conceptual issues in the Service Composition Layer of a SOA, when following a process-driven approach to services composition. 


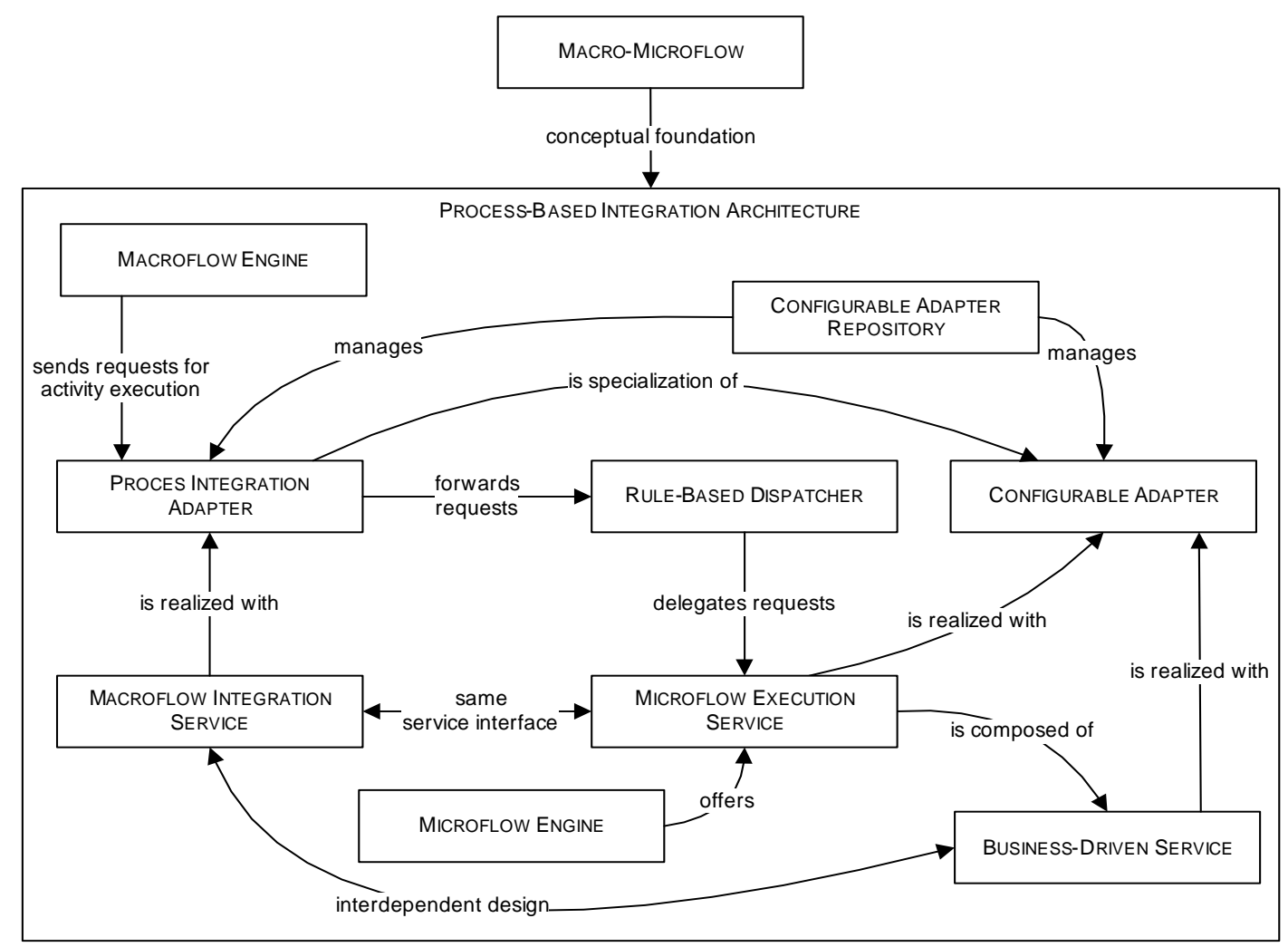

Figure 5. Overview: Pattern language for process-oriented integration of services

The patterns and pattern relationships for designing a Service Composition Layer are shown in Figure 5. In the pattern language, the pattern MACRO-MICROFLOW sets the scene and lays out the conceptual basis to the overall architecture. The pattern divides the flow models into so-called macroflows, which describe the long-running business processes, and microflows, which describe the short-running technical processes.

The PROCESS-BASED INTEGRATION ARCHITECTURE pattern describes how to design an architecture based on sub-layers for the Service Composition Layer, which is following the MACRO-MICROFLOW conceptual pattern.

The remaining patterns in the pattern language provide detailed guidelines for the design of a PROCESS-BASED INTEGRATION ARCHITECTURE. In Figure 5 they are thus displayed within the boundaries of the PROCESS-BASED INTEGRATION ARCHITECTURE pattern.

The automatic functions required by macroflow activities from external systems are designed and exposed as dedicated MACROFLOW INTEGRATION SERVICES. PROCESS INTEGRATION ADAPTERS connect the specific interface and technology of the process engine to an integrated system. A RULE-BASED DISPATCHER picks up the (macroflow) activity execution requests and dynamically decides based on (business) rules, where and when a (macroflow) activity is executed. A CONFIGURABLE ADAPTER connects to another system in a way that allows to easily maintain the connections, considering that interfaces may change over time. A CONFIGURABLE ADAPTER REPOSITORY manages CONFIGURABLE ADAPTERS as components, such that they can be modified at runtime without affecting the systems sending requests to the adapters. A MICROFLOW EXECUTION SERVICE abstracts the technology specific API of the 
MICROFLOW ENGINE and encapsulates the functionality of the microflow as a service. A MACROFLOW ENGINE allows for configuring business processes by flexibly orchestrating execution of macroflow activities and the related business functions. A MICROFLOW ENGINE allows for configuring microflows by flexibly orchestrating execution of microflow activities and the related BUSINESS-DRIVEN SERVICES. To define BUSINESS-DRIVEN SERVICES, highlevel business goals are mapped to to-be macroflow business process models that fulfill these goals and more fine grained business goals are mapped to activities within these processes.

Figure 6 shows an exemplary configuration of a PROCESS-BASED INTEGRATION ARCHITECTURE, in which multiple macroflow engines execute the macroflows. Process-integration adapters are used to integrate the macroflows with technical aspects. A dispatching layer enables scalability by dispatching onto a number of microflow engines. Business application adapters connect to backends.

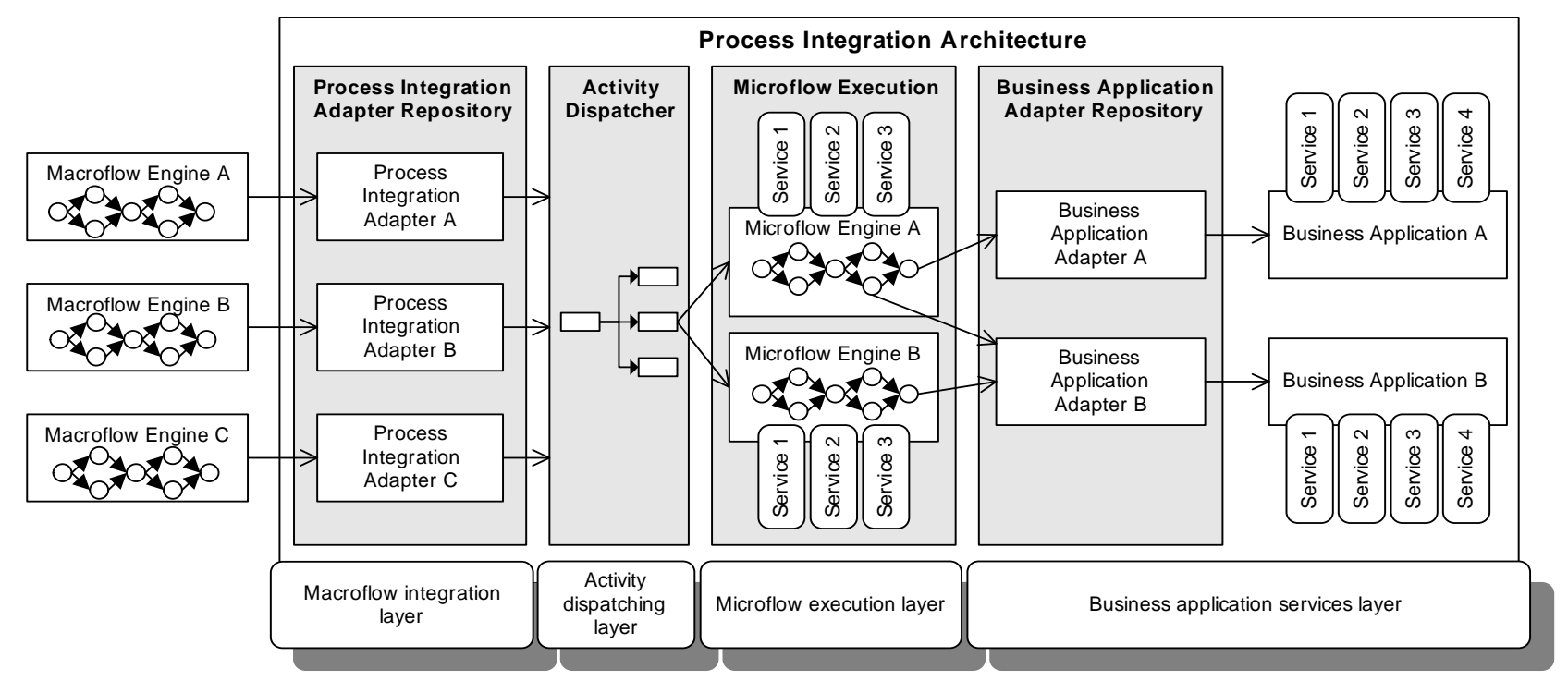

Figure 6. Example Configuration of a Process-based Integration Architecture

\subsection{Pattern primitives for Process/Service Integration}

In this section, we present the pattern primitives for flow abstractions that we have mined from the pattern language in Figure 5. We will concentrate only on one example primitive; the other flow abstraction primitives are summarized in Table 1.

Each primitive is formally defined in the context of the UML2 meta-model using OCL constraints. To illustrate the formal definition of the primitives let us consider the Macro-Microflow Refinement Primitive. This primitive models the situation that Microflow Models are allowed to refine Macroflow Models. In addition to macroflows and microflows, we must consider the Macroflow Steps and Microflow Steps models, introduced by the Process Flow Steps primitive: A process activity node in a macroflow or microflow can optionally be refined by a number of sequential steps that detail the steps performed to realize the process activity. 


\begin{tabular}{|c|c|c|}
\hline Primitive Name & Description & Modeling Solution \\
\hline $\begin{array}{l}\text { Process Flow } \\
\text { Refinement }\end{array}$ & $\begin{array}{l}\text { A macroflow or microflow is refined us- } \\
\text { ing another process flow. }\end{array}$ & $\begin{array}{l}\text { The Activity metaclass is extended with the stereotype ProcessFlowRefinement, } \\
\text { which also introduces tagged values for identifying the refinement. }\end{array}$ \\
\hline $\begin{array}{l}\text { Process Flow } \\
\text { Steps }\end{array}$ & $\begin{array}{l}\text { A macroflow or microflow is refined by } \\
\text { a number of sequential steps. }\end{array}$ & $\begin{array}{l}\text { A specialization of the ProcessFlowRefinement stereotype, called ProcessFlow- } \\
\text { Steps, is introduced and constrained to be a strictly sequential flow. }\end{array}$ \\
\hline $\begin{array}{l}\text { Macroflow } \\
\text { Model }\end{array}$ & $\begin{array}{l}\text { A macroflow can be refined by other } \\
\text { macroflows or macroflow steps. }\end{array}$ & $\begin{array}{l}\text { Macroflows are modeled by a ProcessFlowRefinement stereotype, called } \\
\text { Macroflow, and macroflow steps are modeled as a specialization of ProcessFlow- } \\
\text { Steps, called MacroflowSteps. }\end{array}$ \\
\hline $\begin{array}{l}\text { Microflow } \\
\text { Model }\end{array}$ & $\begin{array}{l}\text { A microflow can be refined by other mi- } \\
\text { croflows or microflow steps. }\end{array}$ & $\begin{array}{l}\text { The microflow model is modeled analogous to the Macroflow Model primitive: } \\
\text { The Microflow and MicroflowSteps stereotypes are introduced. }\end{array}$ \\
\hline $\begin{array}{l}\text { Macro-Micro- } \\
\text { flow Refinement }\end{array}$ & $\begin{array}{l}\text { Microflow Models are allowed to refine } \\
\text { Macroflow Models. }\end{array}$ & $\begin{array}{l}\text { The Microflow Model primitive is extended: If refinedActivityNode of a Mi- } \\
\text { croflow is not empty, the Microflow is a refinement of a Microflow, a Macroflow, } \\
\text { or MacroflowSteps. }\end{array}$ \\
\hline $\begin{array}{ll}\text { Restricted } & \text { Ma- } \\
\text { croflow } & \text { Step } \\
\text { Types } & \end{array}$ & $\begin{array}{l}\text { Macroflow Steps are restricted to } 3 \\
\text { kinds: process function invocation, pro- } \\
\text { cess control data access, and process re- } \\
\text { source access. }\end{array}$ & $\begin{array}{l}\text { Respective stereotypes for the ActivityNode metaclass are introduced: InvokePro- } \\
\text { cessFunction, AccessControlDataItem, and AccessProcessResource. The stereo- } \\
\text { types introduce tagged values for identifying the data item or resource that is ac- } \\
\text { cessed. }\end{array}$ \\
\hline $\begin{array}{ll}\text { Restricted } & \text { Mi- } \\
\text { croflow } & \text { Step } \\
\text { Types } & \end{array}$ & $\begin{array}{l}\text { Microflow Steps are restricted to } 2 \\
\text { kinds: process functions invocations } \\
\text { and process control data access. }\end{array}$ & $\begin{array}{l}\text { This primitive can be modeled using } 2 \text { stereotypes for the ActivityNode metaclass: } \\
\text { InvokeProcessFunction and AccessControlDataItem. }\end{array}$ \\
\hline $\begin{array}{l}\text { Synchronous } \\
\text { Service Invoca- } \\
\text { tion }\end{array}$ & A service is invoked synchronously. & $\begin{array}{l}\text { The SyncServiceInvocation, GetInvocationData, and WriteServiceResult stereo- } \\
\text { types extend the Activity Node metaclass. }\end{array}$ \\
\hline $\begin{array}{l}\text { Asynchronous } \\
\text { Service Invoca- } \\
\text { tion }\end{array}$ & A service is invoked asynchronously. & $\begin{array}{l}\text { The AsyncServiceInvocation and GetInvocationData stereotypes extend the Activ- } \\
\text { ity Node metaclass. An asynchronous service invocation might have no result. }\end{array}$ \\
\hline $\begin{array}{l}\text { Fire and Forget } \\
\text { Invocation }\end{array}$ & $\begin{array}{l}\text { A service is invoked asynchronously } \\
\text { with fire and forget semantics. }\end{array}$ & $\begin{array}{l}\text { The stereotype FireAndForgetInvocation specializes the AsyncServiceInvocation } \\
\text { stereotype. No result is written for this service. }\end{array}$ \\
\hline $\begin{array}{l}\text { One Reply } \\
\text { Asynchronous } \\
\text { Invocation }\end{array}$ & $\begin{array}{l}\text { A service is invoked asynchronously, } \\
\text { and exactly one result is coming back. }\end{array}$ & $\begin{array}{l}\text { The OneReplyInvocation stereotype specializes AsyncServiceInvocation. We can- } \\
\text { not guarantee that the result comes back in the same Activity, but there must be } \\
\text { exactly one result for a OneReplyInvocation. }\end{array}$ \\
\hline $\begin{array}{l}\text { Multiple Reply } \\
\text { Asynchronous } \\
\text { Invocation }\end{array}$ & $\begin{array}{l}\text { A service is invoked asynchronously, } \\
\text { and multiple results are coming back. }\end{array}$ & $\begin{array}{l}\text { The MultipleReplyInvocation stereotype specializes AsyncServiceInvocation. We } \\
\text { cannot guarantee that the result comes back in the same Activity, but there must be } \\
\text { at least one result reception. }\end{array}$ \\
\hline $\begin{array}{l}\text { Process Control } \\
\text { Data Driven In- } \\
\text { vocation }\end{array}$ & $\begin{array}{l}\text { A service is invoked using only data } \\
\text { from the process control data. }\end{array}$ & $\begin{array}{l}\text { A service invocation is modeled by a refined Activity Node stereotyped as Process- } \\
\text { ControlDataDrivenInvocation. This activity must contain a ServiceInvocation, and } \\
\text { ReadServiceData/WriteServiceResult must be used which specialize AccessPro- } \\
\text { cessControlData. }\end{array}$ \\
\hline
\end{tabular}

Table 1. Flow Abstraction Pattern Primitives Overview 


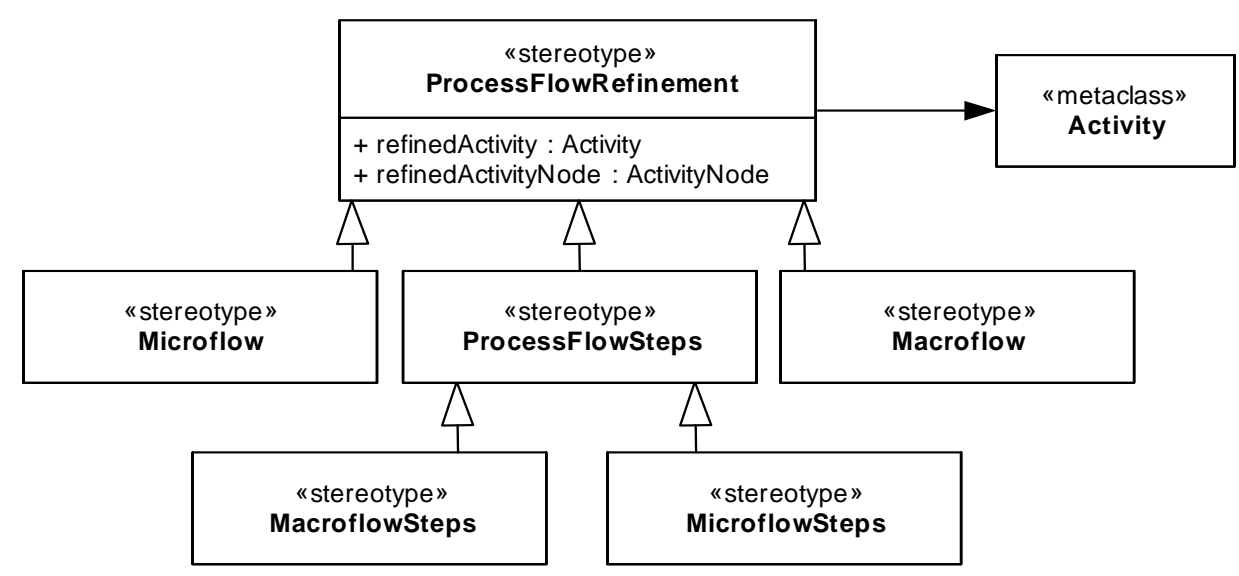

Figure 7. Macro-/Microflow: Stereotypes for the Primitives

To model this primitive, we first must introduce UML2 stereotypes to distinguish the different kinds of refined/refinement activities (see Figure 7) in the UML2 models. The same extension for the Activity meta-class looks as follows in the Frag textual syntax:

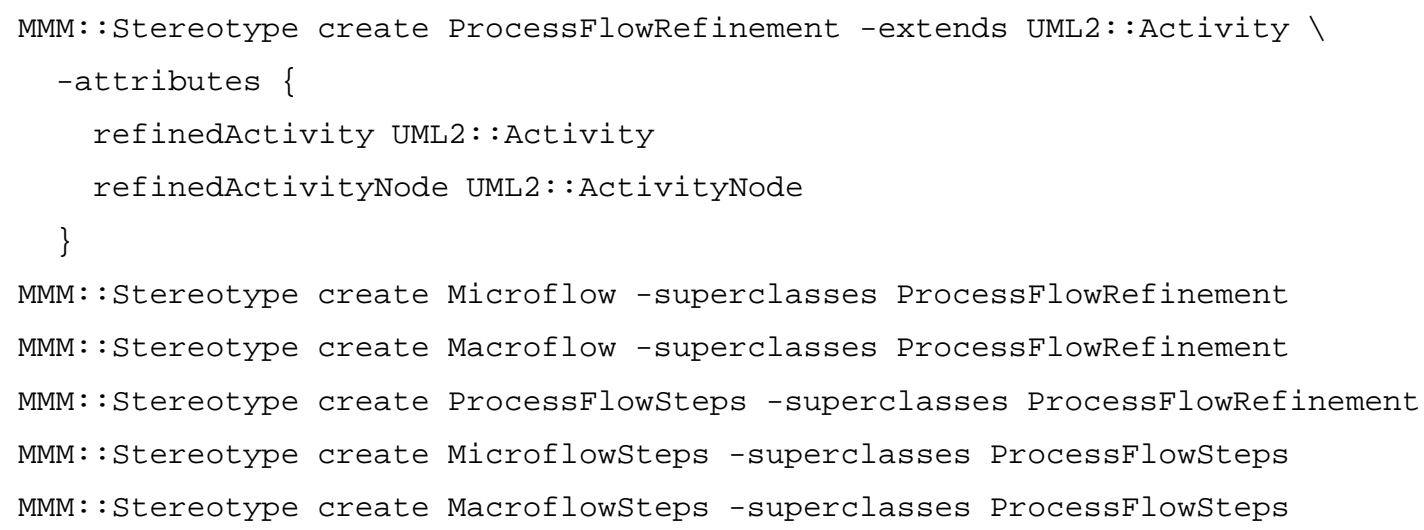

We can model the Macro-Microflow Refinement primitive by constraining the Microflow Activities. In particular, if the refinedActivityNode and refinedActivity tag values of a Microflow are not empty, the Microflow is a refinement of another Microflow, a Macroflow, or a MacroflowSteps Activity. This can be formally modeled using the following OCL constraint:

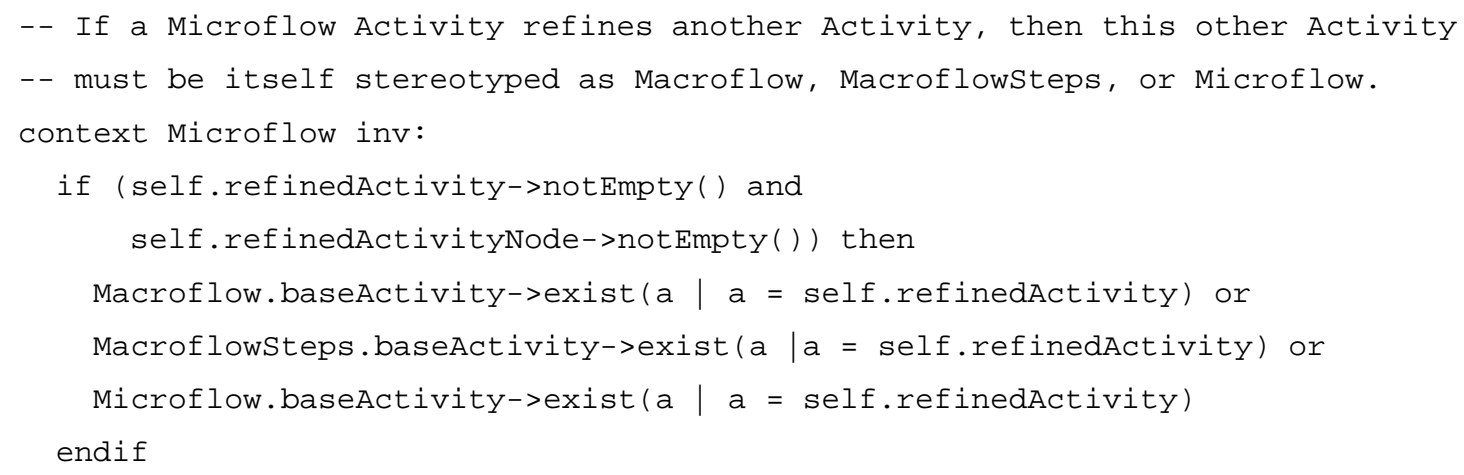

The syntax of the FCL constraints, expressed in Frag, have a slightly different syntax than OCL, but are semantically equivalent (i.e. the constraints can be automatically translated between OCL and FCL). Here is the same 
invariant in the FCL syntax:

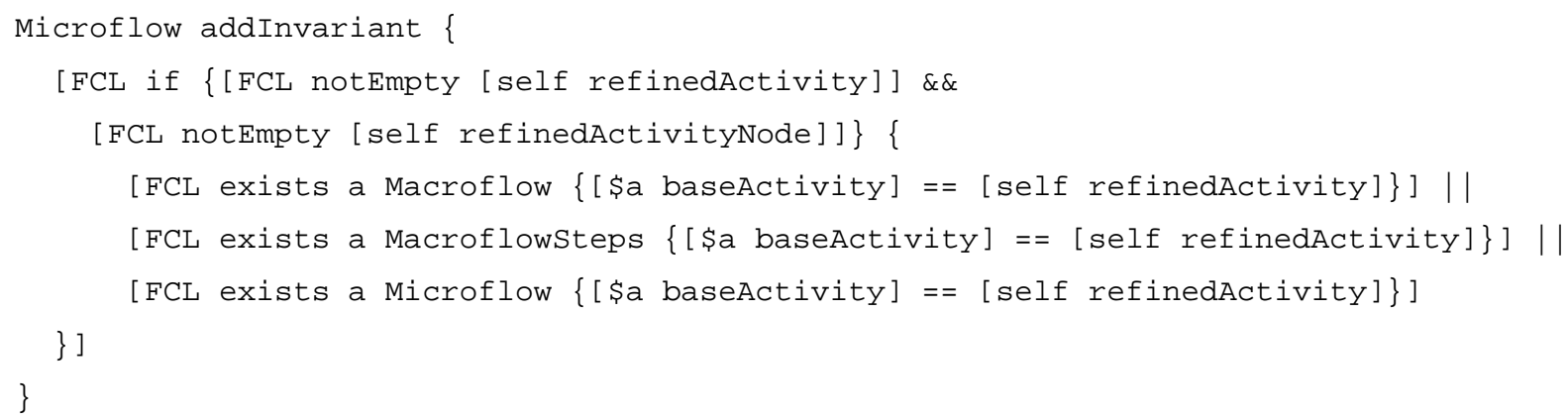

The task of such constraints is basically to limit the use of the primitives to the acceptable parameters - following the pattern descriptions in which the primitives are used - but no further. For each primitive we have hence described all such formal constraints (the others are omitted here for space reasons). Thus each primitive describes a formal, parameterizable building block that can be used in the solution of the patterns.

\subsection{Modeling patterns using the pattern primitives for process-oriented integration of services}

The formal, parameterizable building blocks represented by the pattern primitives are not yet linked to the patterns. The patterns cannot be themselves formally modeled, but we can identify the pattern primitives that occur in individual patterns. For instance, some of the primitives are mandatory in a pattern, others are optional, still others are only used in specific variants, etc. This mapping of patterns to pattern primitives hence provides us with modeling constructs that can be differently combined for different pattern instances, but must conform to the pattern-to-primitive mapping. If a primitive is used in a pattern instance, all formal constraints of the primitive must be fulfilled. Hence, the primitives formally encode the proven practices documented in the patterns as modeling constructs.

In the remainder of this section, we illustrate our approach using the example of modeling the MACROMICROFLOW pattern. The other patterns are modeled following the same basic approach. Especially we want to model that the pattern structures a process model into two kinds of processes, macroflow and microflow. The pattern strictly separates the macroflow from the microflow, and uses the microflow only for refinements of the macroflow activities.

Both in macroflows and microflows we can observe refinements. The different kinds of refinement can be modeled using the Process Flow Refinement primitive. Process Flow Refinement is a generic primitive that can be used for modeling all kinds of process refinements.

The following code illustrates how the Frag textual DSL syntax can be used to describe a model (we only show the excerpt for Model1 from Figure 8):

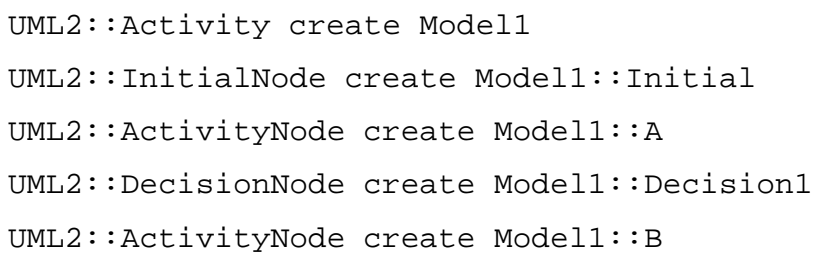




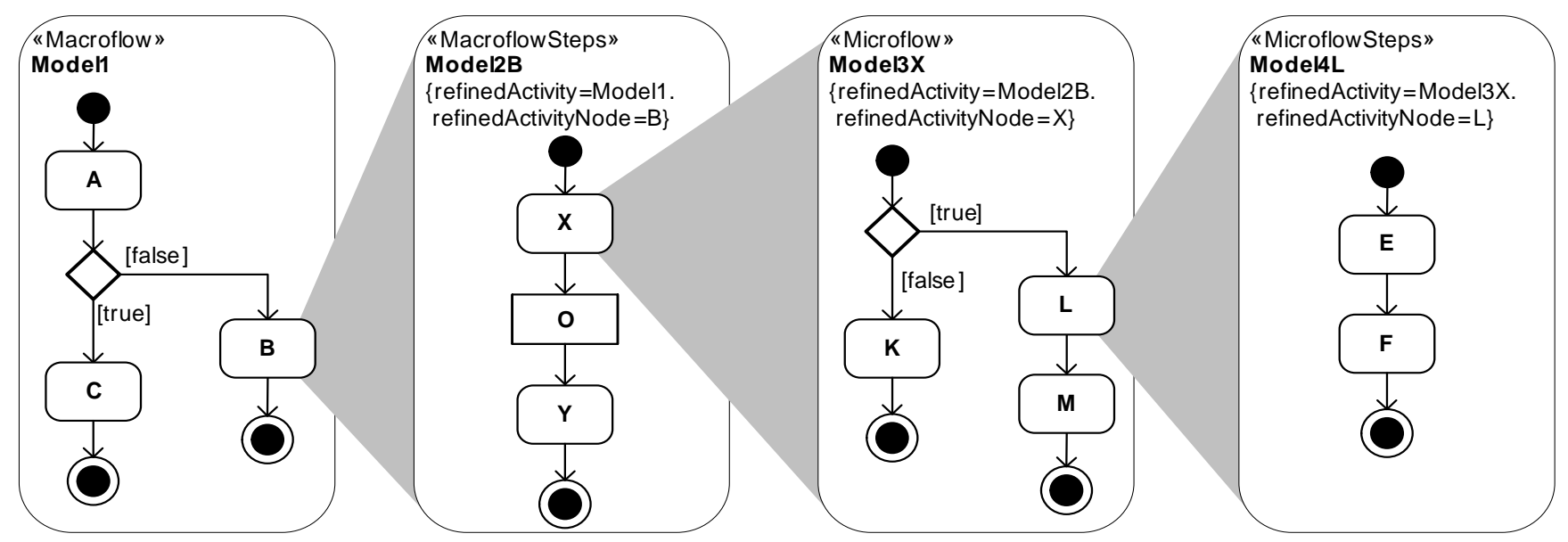

Figure 8. Macro-Microflow modeling example 1

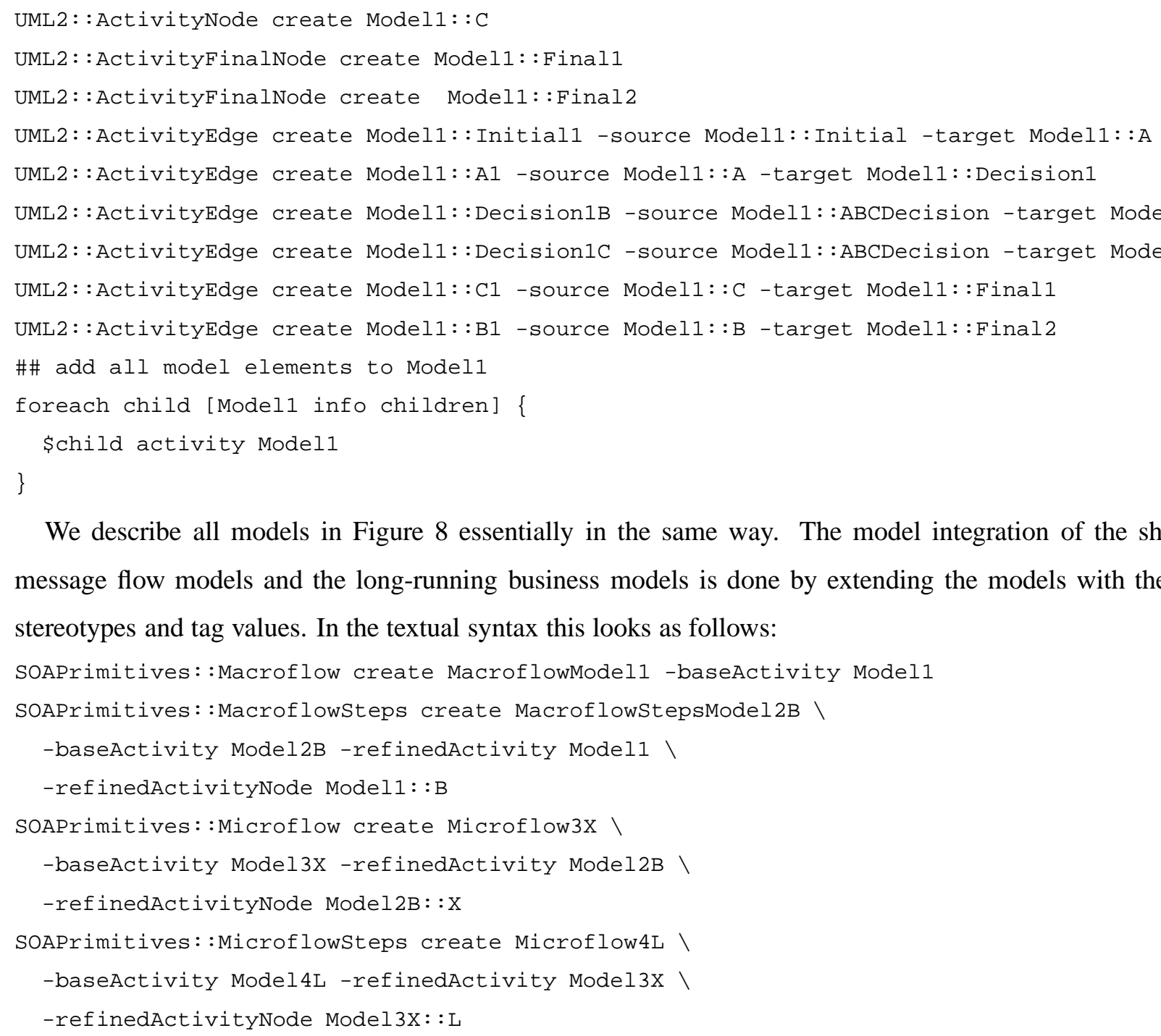
stereotypes and tag values. In the textual syntax this looks as follows:

We describe all models in Figure 8 essentially in the same way. The model integration of the short-running message flow models and the long-running business models is done by extending the models with the respective

After these stereotypes have been defined, the OCL constraints of the primitives enforce that those four models can only be composed in a way that is valid according to the Macro-Microflow Refinement primitive. Figure 8 hence 
shows a model conforming to the constraints.

For the MACRO-MICROFLOW pattern it is mandatory that the Macro-Microflow Refinement primitive is used, and at least one Macroflow Model and one Microflow Model with a refinement relationship between them must be present in a model. There are different specific kinds of refinement possible:

- Macroflows can be refined by other macroflows. That is, a Macroflow Model refines another Macroflow Model.

- Microflows can be refined by other microflows. That is, a Microflow Model refines another Microflow Model.

- Macroflows can be refined by microflows. That is, a Microflow Model refines another Macroflow Model.

- Often it is additionally possible to refine each activity in a macroflow or microflow using a sequence of activity steps. This can also be modeled using the Process Flow Steps primitive, either at the Macroflow Model or Microflow Model level.

The Macro-Microflow Refinement primitives allows us to model a number of pattern variants of the MACROMICROFLOW pattern. For instance, the MACRO-MICROFLOW structure may strictly follow a refinement in macroflow $\rightarrow$ macroflow steps $\rightarrow$ microflow $\rightarrow$ microflow steps. Figure 8 shows an example of such a refinement using the UML2 representations of the pattern primitives.

Another, different exemplary structure is that the macroflows at the highest level depict the main business processes, which are then refined by other macroflows depicting sub-processes that are still business-oriented. These macroflows are refined stepwise via other macroflows. Finally, the macroflow activities of the lowest granularity are refined by microflows. That is, in this second example, there are no process flow steps used in the model, but multiple refinements at the macroflow level. Figure 9 shows an example of such a refinement using the UML2 representations of the pattern primitives.

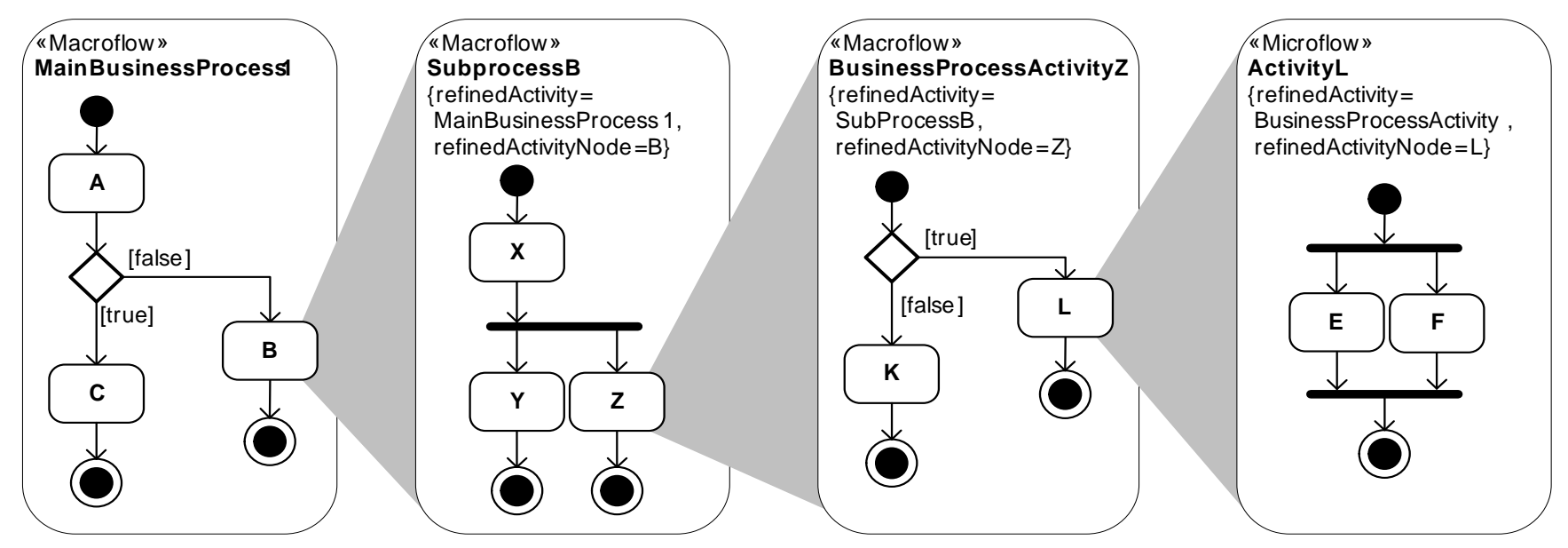

Figure 9. Macro-Microflow modeling example 2

Numerous other variants of the MACRO-MICROFLOW pattern are possible and can hence be modeled with the primitives introduced in the previous section. Please note that the flexibility of model assembly through primitives is 
a very important characteristic of our approach, because it enables us to represent the inherent variability of software pattern solutions.

\section{Modeling Architectural Abstractions in Process-Driven Integration Of Services}

As seen above, the MACRO-MICROFLOW pattern has implications for short-running message flow models and the long-running business models, and we were able to integrate the two model types (and even add macroflow/microflow steps as an another kind of modeling abstraction). In our approach, these two kinds of models are modeled with the same model type: activity diagrams. The patterns, however, also have implications for other model types, such as the architectural components in the system or business object models. To model those abstractions, we additionally need to consider architectural abstractions and object-oriented design abstractions.

In UML, business objects can be modeled using class diagrams. Architectural abstractions can be modeled via component diagrams. Component diagrams are a specialization of class diagrams. Therefore, our approach for integrating those models with flow models is very similar for both class diagrams and component diagrams. Hence, we demonstrate our approach only for one of those abstractions in depth: architectural components.

Our approach is to first find suitable pattern primitives for modeling the architectural abstraction. If further integration is needed, we try to model the flow model and architectural model in parallel, especially with overlapping constraints. If such a "loose" integration is not enough, we model primitives and constraints that cut across model boundaries. In the following sub-sections, we will demonstrate each of these solutions in turn.

\subsection{Modeling Architectural Abstractions with Pattern Primitives}

Architectural modeling with pattern primitives follows the same approach as introduced for the flow abstractions. In the context of architectural abstraction, we have introduced similar primitives for architectural patterns (see [34]). Let us consider the PROCESS-BASED INTEGRATION ARCHITECTURE pattern and the Callback primitive as an example.

In the PROCESS-BASED INTEGRATION ARCHITECTURE pattern, different kinds of components are connected. Figure 6 shows an exemplary larger configuration, in which multiple macro-/microflow engines and a dispatcher are used. Of course, there are also significantly smaller PROCESS-BASED INTEGRATION ARCHITECTURE configurations. For instance, a single macroflow engine can interact directly with a single microflow engine, which connects to backends via service-based adapters. The modeling support for the PROCESS-BASED INTEGRATION ARCHITECTURE pattern should allow for flexibly assembling different kinds of PROCESS-BASED INTEGRATION ARCHITECTURE models. In the PROCESS-BASED INTEGRATION ARCHITECTURE this flexibility is achieved by following asynchronous messaging patterns from [15].

The Callback primitive [34] can be used to model the reactive behavior in these patterns: A callback denotes an invocation to a component $B$ that is stored as an invocation reference in a component $A$. The callback invocation is 
executed later, upon a specified set of runtime events. Between two components $A$ and $B$, a set of callbacks can be defined. To capture the semantics of callbacks properly in UML, we propose five stereotypes:

- IEvent: A stereotype that extends the 'Interface' metaclass and contains a number of methods that are exclusively trigger events for a callback.

- ICallback: A stereotype that extends the 'Interface' metaclass and contains a number of methods that serve exclusively as callback methods.

- EventPort: A stereotype that extends the 'Port' metaclass and is typed by two interfaces: IEvent as a provided interface and ICallback as a required interface.

- CallbackPort: A stereotype that extends the 'Port' metaclass and is typed by two interfaces: ICallback as a provided interface and IEvent as a required interface.

- Callback: A stereotype that extends the 'Connector' metaclass and specifies the semantics of a callback connector, which connects an EventPort of a component to a matching CallbackPort of another component.

Again, we have formalized the constraints using OCL (see [34]), and defined the meta-model and constraints in Frag, so that we can use the constraints on components that represent the PROCESS-BASED INTEGRATION ARCHITECTURE components.

All components in a PROCESS-BASED INTEGRATION ARCHITECTURE are interconnected following the callback style because they use asynchronous communication. The event ports of each layer are listening to events from the higher-level layer, and when an event arrives, they call into the lower-level layer. Once a result is received, it is propagated back into the higher-level layer using a Callback. Figure 10 shows an example UML2 model for a Callback configuration modeling the situation from Figure 6 in a more formal way.

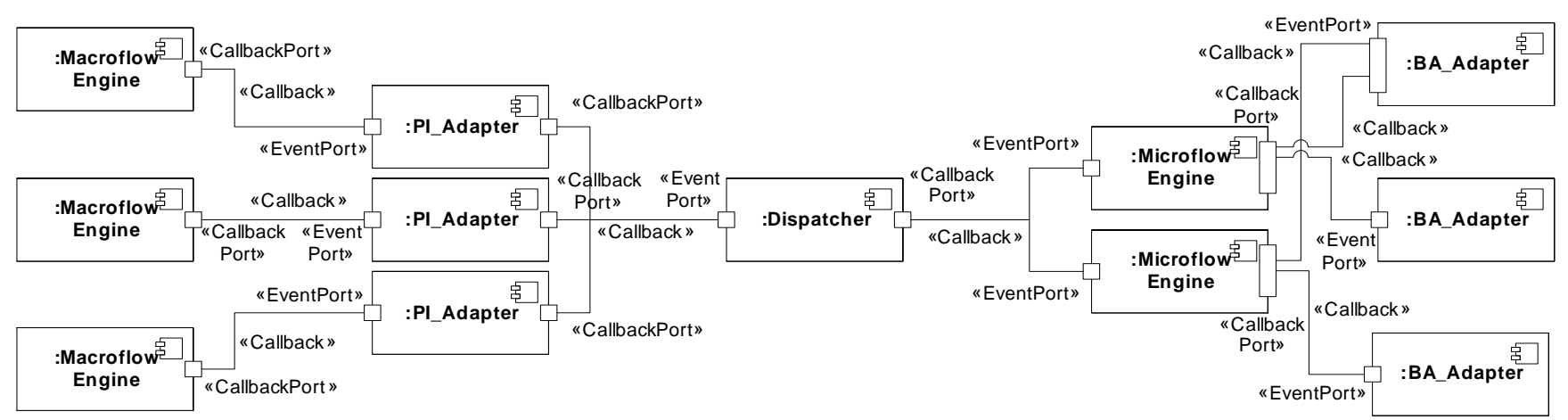

Figure 10. UML2 Model for the Example Configuration 


\subsection{Integrating Architectural and Flow Abstraction Models by Modeling in Parallel}

In addition to the architectural flexibility of the PROCESS-BASED INTEGRATION ARCHITECTURE pattern, we need to model the pattern's constraints. If the pattern implementation follows the MACRO-MICROFLOW pattern, analogous constraints to the macro-microflow refinement in the flow models must be introduced, such as: components that represent the microflow should not invoke macroflow functionality, macroflow adapters should not be used at the microflow level and vice versa, the dispatcher should only invoke short running microflows, etc. Such issues can be modeled "in parallel" to the flow model. That is, we do not need primitives or constraints that cut across modeling domains.

In this situation, we can use another architectural primitive from [34]: Layering. Layering describes groups of components and further constrains them. Specifically, it entails that group members from layer $X$ may call into layer $X-1$ and components outside the layers, but not into layer $X-2$ and below. To model Layering in UML2, we introduce the Layer stereotype, which specializes the Group stereotype (which itself is an extension of the Package metaclass). We also impose the following constraints: a component can only be member of one layer and not multiple layers; components who are members of layer $X$ may call their fellow components in layer $X$, as well as components in layer $X-1$ but not in other layers (e.g. $X-2$ and below). It is noted that there is no constraint about calling components in layer $X+1$ or above, since this is a specific issue to the pattern realization. Also, we introduce the tag definition layerNumber for Layers which represents the number of the layer in the ordered structure of layers. Figure 11 shows an UML2 model that extends the model from Figure 10 using the Layering primitive.
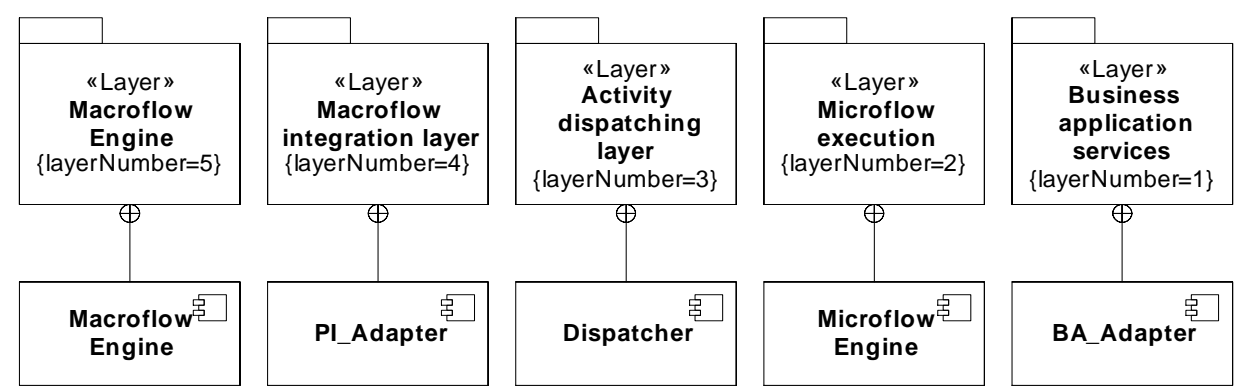

Figure 11. Extending the Example Configuration with Layering

In this example, we have modeled the integration of the macroflow-microflow refinements at the flow model level with the architectural components by introducing OCL constraints for the refinements between the flow models, and by adding similar constraints to the architectural model. That is, the primitive Macro-Microflow Refinement was used at the flow model level and Callback/Layering were used at the architectural level. Sometimes this is not enough, and it is necessary to extend this strategy and add a direct relationship between the flow models and the architectural models. Then the solution described in the next section should be used. 


\subsection{Integrating Architectural and Flow Abstraction Models Using Cross-Model Primitives}

In some cases it is necessary to introduce a formal dependency between different model types to model a concern properly. For instance, to correlate the events and callbacks between the components, we usually pass a CORRELATION IDENTIFIER [15] between the components. In the component model, we need to model which components use which CORRELATION IDENTIFIER because in a PROCESS-BASED INTEGRATION ARCHITECTURE multiple CORRELATION IDENTIFIERS can be used. In addition, we must ensure that the macroflow and microflow models pass a valid CORRELATION IDENTIFIER type to all asynchronous invocations.

To model this situation, we first introduce a new architectural primitive, Correlation. Like Layering, the Correlation primitive also extends the Grouping primitive from [34]. It introduces two stereotypes:

- CorrelationIdentifier: A stereotype that extends the 'Class' metaclass and contains a tag value correlationGroup of the type Package.

- CorrelationGroup: A subclass of the Group stereotype which extends the 'Package' metaclass.

There are the following constraints: A correlation group package, used in the tag value correlationGroup, must be stereotyped as CorrelationGroup. Each correlation group must have a CorrelationIdentifier with a correlationGroup tag value that points to it.

Using this primitive we can define that certain components belong to a correlation group and use a specific class (or component) as correlation identifier. For instance, the component model in Figure 12 expresses that the components MacroflowEngine, PI_Adapter, and Dispatcher form a correlation group with the correlation identifier C_ID.
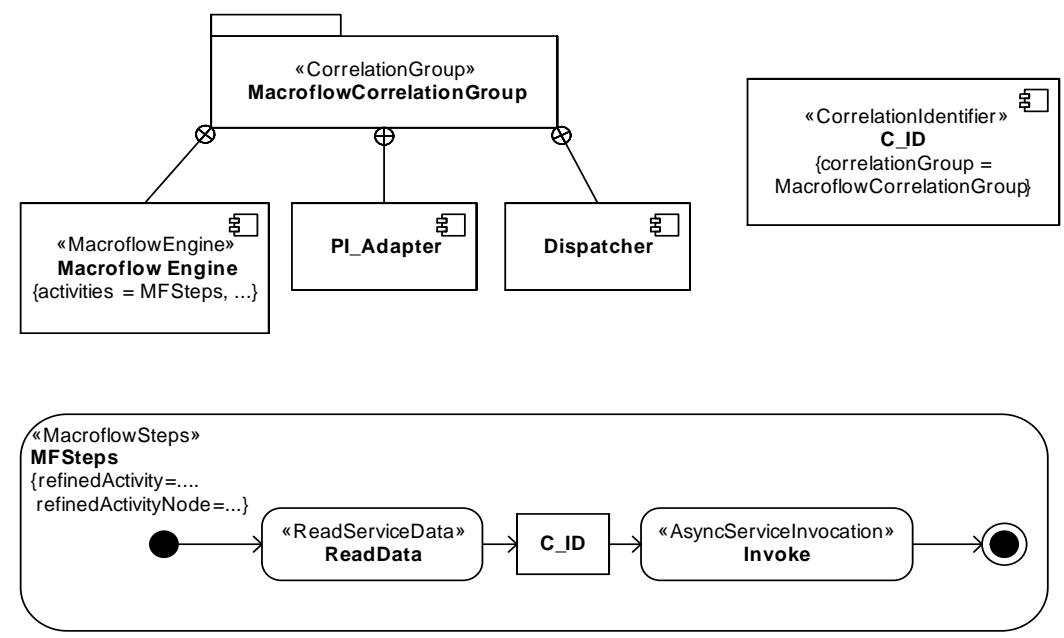

Figure 12. Correlation Identifier Group

To model the dependency to the macroflow/microflow models we introduce two new primitives for modeling the patterns MACROFLOW ENGINE and MICROFLOW ENGINE, which are part of the PROCESS-BASED INTEGRATION ARCHITECTURE. The goal is to be able to model which macroflow models are executed on which MACROFLOW 
ENGINE and which microflow models are executed on which MICROFLOW ENGINE. We introduce three new stereotypes:

- ProcessEngine: A stereotype that extends the 'Component' metaclass and contains a tag value activities of the type Activity.

- MacroflowEngine: A stereotype that subclasses ProcessEngine to specify a component that executes macroflows.

- MicroflowEngine: A stereotype that subclasses ProcessEngine to specify a component that executes microflows.

There is are OCL constraints that specify that a MacroflowEngine can only have activities that are stereotyped either as Macroflow or MacroflowSteps. A MicroflowEngine can only have activities that are stereotyped either as Microflow or MicroflowSteps. That is, using these primitives and the constraints, we have introduced a formal dependency between the component and flow models.

Finally, we need to specify the correlation constraints as an OCL constraint:

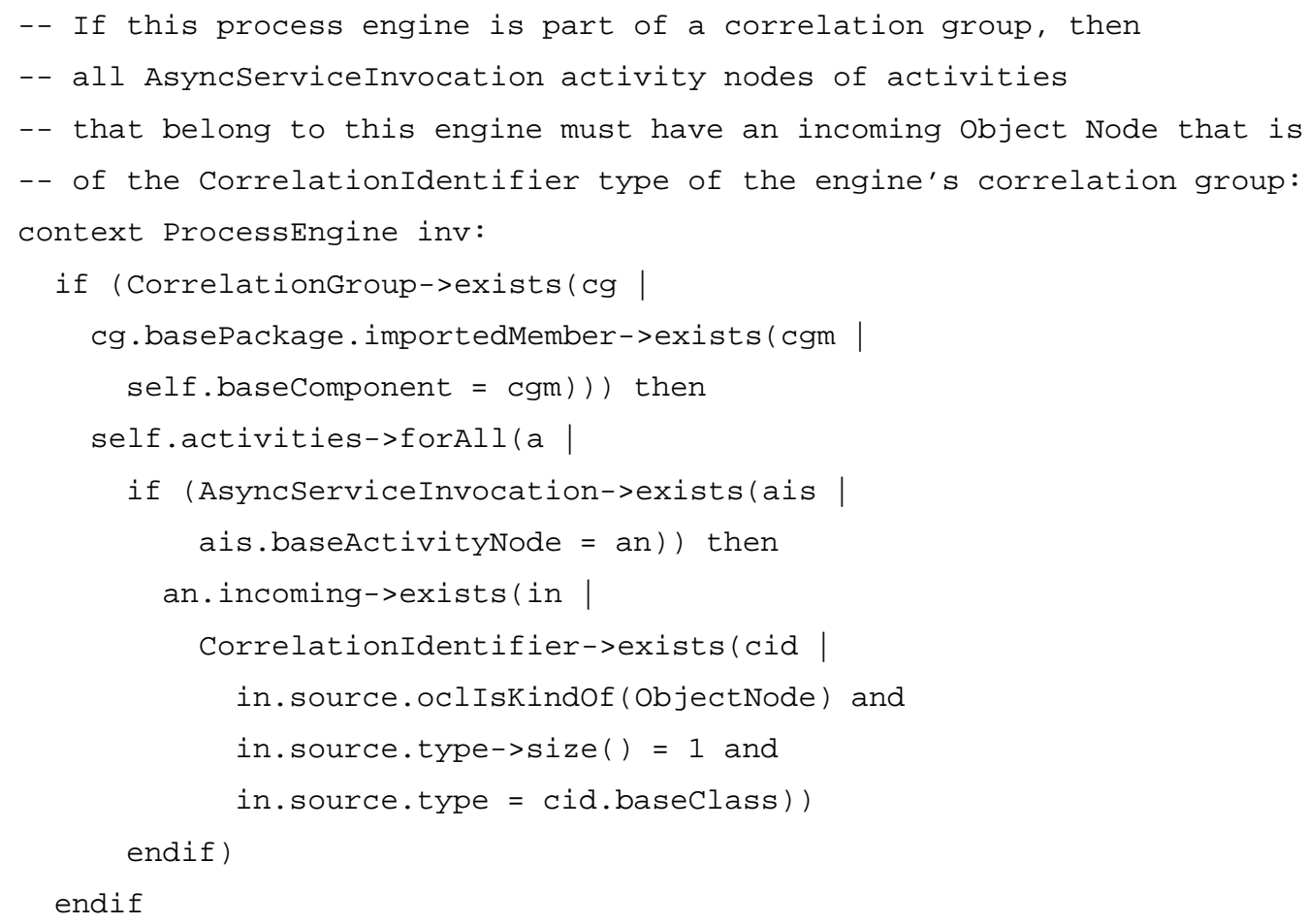

Now we have ensured that all asynchronous invocations in a correlation group's activities - at the macroflow, macroflow steps, microflow, and microflow steps level - must have a correlation identifier type of the correlation group as an incoming object node in the activity diagram. An example activity for the macroflow correlation group example is also shown in Figure 12. 


\section{Related Work}

A number of other approaches for the documentation of SOA proven practices are available. For instance, a number of reference architectures and blueprints have been proposed [27, 5, 28]. These approaches focus on specific technologies and apart from this specific focus neither modeling support nor tool support that is more universally applicable has been proposed.

Zimmermann et al. present a generic modeling approach for SOA projects [36]. As in our approach, the approach by Zimmermann et al. is based on project experiences and distills proven practices from practical cases. The approach also integrates multiple kinds of models for a SOA:

- object-oriented analysis and design,

- enterprise architecture, and

- business processes.

Even though there are many similarities to our approach, there is the difference that the authors use rather informal models to illustrate their approach and do not provide a concept for explaining the conceptual building blocks of the architecture (like the patterns in our approach). They do not provide a concept for using the approach in the context of MDSD.

Some other approaches define particular aspects of service or business process composition using formal specifications, such as activity-based finite automata [11] or interval temporal logic [24]. Desai et al. [7] propose to abstract business processes using interaction protocol components which represent an abstract, modular, publishable specification of an interaction among different partner roles in a business process. In general, however, these approaches in the first place aim to support verifiability, and hence can only model specific aspects of service composition. Our approach aims for a significantly extended scope: general purpose modeling of process-driven SOAs based on a formalization of proven practices and model-driven tool support.

Some approaches use the Semantic Web to model the integration of (Web) services and process. OWL-S [6] includes a process model for Web services and it uses semantic annotations to enable dynamic composition. Cardoso and Sheth [4] use Semantic Web ontologies to facilitate the interoperability of heterogeneous Web services in the context of workflows. This is a different - though not contradictory - approach to model the integration of services and processes than the process flow paradigm used in our approach.

There are many modeling approaches for business processes. Two out of many examples are Event-Driven Process Chains (EPC) [17] and the BPMN [21]. Both approaches are related to our approach because they focus on one (of the many) modeling aspects of process-driven SOAs, business process modeling. Our approach has in common with these approaches that we use the flow abstraction as the central modeling abstraction. Unlike the BPMN, our approach is based on a formal meta-model. Among others, Kindler has proposed formal semantics for EPCs [18]. In contrast to modeling approaches for business processes, our approach allows to integrate other models covering 
aspects of software design, software architectures, messages flows, etc. of a process-driven SOA. That is, we take a more "integrative" view than those more specific approaches.

Business process management tools, such as Adonis [1] or Aris [16], describe a holistic model of business process management, ranging from strategic decisions to the design of business processes. They also provide mappings (imports/exports) to the realization and execution of processes. They are integrated with standard model types and extensible with new model types. Such tools are related to our approach at a high level because they represent important prior art in the field of model integration. But they do not - such as our approach - concentrate on models based on proven practices. They also do not specifically focus on the field of process-driven SOAs; they are more focused on the business processes. However, an extensible tool suite like Adonis can be used for providing input models for our approach or be extended to model the DSLs in our approach.

The workflow patterns [2] describe concepts of workflow languages. They are conceptually closer to our notion of pattern primitives than to the pattern concept as it is used in the pattern community. That is, the workflow patterns are formalizable constructs (e.g., formalized in the Petri-net-based language YAWL). The general approach to formalize pattern primitives using (colored) Petri nets is also applicable to our approach, e.g., if validation or verification is the goal. We use a formalizable subset of the UML and OCL instead because our goals are a general modeling support, model validation, and to formally integrate different models of a SOA.

Our approach extends the MDSD concept proposed for instance in $[26,12]$ with the idea to use one common metameta-model for model integration, pattern primitives as modeling constructs based on proven practices, and model validation tools for these concepts. In Chapter 13 of the book Software Factories [12] it is briefly discussed how typical MDSD concepts can be used to support SOA modeling, but only with a focus on Web Services technology. Essentially, the process description, e.g. in BPEL, is seen as a platform for implementing abstractions in a product line, and the services are seen as product line assets for systematic reuse. This view does not contradict our approach, but our approach goes beyond this vision. Through the common meta-meta-model we can integrate any kind of model types; hence, process descriptions are not only a platform, but a first-class model type. In the software factories approach, it is advised that patterns are used as proven practices, but there is no guidance how to map them to formal modeling constructs, like the pattern primitives in our approach. Of course, any MDSD approach can be extended to support our approach.

There are a number of UML profiles for various SOA aspects. Wada et al. [31] propose an UML profile to model non-functional aspects of SOAs and present an MDSD tool for generating skeleton code from these models. Heckel et al. [13] propose a UML profile for dynamic service discovery in a SOA by providing stereotypes that specify the relationships among service implementations, service interfaces, and requirements. Gardner et al. [10] define a UML profile to specify service orchestration with the goal to map the specification to BPEL code. Vokac and Glattetre [30] use - as in our examples - UML profiles to define DSLs. The proposed UML profile supports data integration issues. Even though these approaches focus on different application areas of a SOA, they share similar characteristics: In contrast to our approach, they focus on a single type of model, not on model integration or on 
extensibility with other model types. The modeling constructs are not systematically derived from proven practices. Hence, the approaches are very specific for the application area they focus on. By redefining such profiles to conform to a common meta-meta-model they could, however, be integrated into our approach.

There are also a number of related approaches for the formalization of pattern specifications $[8,20,25,19]$. These approaches mainly describe simple single patterns from [9] in a formal manner. But there are a number of issues with these approaches: First, these approaches in first place do only specify a specific implementation variant of these patterns, not all possible variants. That is, they are too limited in the abstractions they propose to grasp the rich concepts found in patterns, and do not do not deal with the inherent variability found in pattern descriptions. Secondly, they do not provide a concept for dealing with the central concept of pattern languages which explain the relationships of different pattern-based design decisions in terms of sequences. We have resolved these problems using the pattern primitive concept and applied our primitives to a pattern language that is (much) more complex than single patterns from [9] - a central prerequisite for a complex domain like process-driven SOAs.

\section{Evaluation}

In this paper, we have introduced a particular combination of features to model process-driven SOAs based on proven practices. In contrast to the related work in this area, we have defined a tool chain for MDSD, which is extensible with domain-specific modeling languages for different model types. We support models for business processes, message flows, OO design, and software architecture - and programming language code/snippets provided as individual code in the MDSD tool chain. Our approach is extensible with new model types, especially domainspecific models. We plan to extend our approach in additional relevant modeling domains, such as organizational models or human-interaction models. None of the related approaches offers sufficient support for all these model types. Except for the approach by Zimmermann et al., Adonis/Aris, and MDSD/software factories, the related work concentrates mostly on one type of modeling domain.

Adonis/Aris provide an explicit extensibility model for model types. MDSD/software factories provide the same extensibility model as our approach: DSLs based on formal meta-models.

Cross-model integration is partially supported by many of the other approaches. However, usually there are less modeling domains supported for specific modeling approaches. The integration is often not defined formally (e.g. through a common meta-meta-models or a constraint language). Some approaches, like BPMN, provide the notion of different kinds of abstractions, but do not introduce multiple modeling views. MDSD/software factories, of course, integrate the models in the code generator, but usually not at the meta-model level. In MDSD/software factories it is generally possible to follow our approach to cross-model integration. A common meta-meta-model is not enforced, though it is common (i.e., to use the model of the generator as a common meta-meta-model).

Our approach is not the only approach that is based on proven practices, but only our approach and the workflow patterns approach combine proven practices and formal models. In YAWL, the workflow patterns are provided as language constructs; hence in the workflow patterns approach the flexibility of assembly of pattern primitives is not 


\begin{tabular}{|c|c|c|c|c|c|c|c|}
\hline & $\begin{array}{l}\text { Modeling } \\
\text { constructs } \\
\text { based on } \\
\text { proven } \\
\text { practices }\end{array}$ & $\begin{array}{l}\text { Formalized } \\
\text { models }\end{array}$ & $\begin{array}{l}\text { Meta- } \\
\text { model } \\
\text { validation }\end{array}$ & $\begin{array}{l}\text { Constraint } \\
\text { validation }\end{array}$ & $\begin{array}{l}\text { Model- } \\
\text { based } \\
\text { verification }\end{array}$ & $\begin{array}{l}\text { Cross- } \\
\text { model } \\
\text { integration }\end{array}$ & $\begin{array}{l}\text { Extensibi- } \\
\text { lity model } \\
\text { for model } \\
\text { types }\end{array}$ \\
\hline $\begin{array}{l}\text { Reference architec- } \\
\text { tures \& blueprints } \\
{[27,5,28]}\end{array}$ & yes & no & no & no & no & partially & no \\
\hline $\begin{array}{l}\text { Approach by Zimmer- } \\
\text { mann et al. }\end{array}$ & yes & no & no & no & no & partially & no \\
\hline $\begin{array}{l}\text { Formal specification } \\
\text { approaches }[11,24]\end{array}$ & no & yes & no & no & yes & no & no \\
\hline $\begin{array}{l}\text { Interaction protocols } \\
{[7]}\end{array}$ & no & yes & no & no & yes & partially & no \\
\hline $\begin{array}{l}\text { Semantic Web Ser- } \\
\text { vices }[6,4]\end{array}$ & no & yes & partially & no & no & partially & no \\
\hline$E P C[17,18]$ & no & yes & no & no & yes & no & no \\
\hline$B P M N[21]$ & no & no & no & no & no & partially & no \\
\hline Adonis [1], Aris [16] & no & no & partially & partially & no & partially & yes \\
\hline $\begin{array}{l}\text { Workflow patterns + } \\
\text { YAWL [2] }\end{array}$ & yes & yes & no & no & yes & no & no \\
\hline $\begin{array}{l}\text { MDSD/Software Fac- } \\
\text { tories }[26,12]\end{array}$ & no & yes & yes & yes & no & partially & yes \\
\hline $\begin{array}{l}\text { UML profiles for SOA } \\
{[31,13,10,30]}\end{array}$ & no & partially & partially & partially & no & no & no \\
\hline $\begin{array}{l}\text { Pattern } \\
\text { based } \\
\text { MDSD for } \\
\text { process-driven SOA }\end{array}$ & yes & yes & yes & yes & no & yes & yes \\
\hline
\end{tabular}

Table 2. Evaluation of the approach 
(yet) supported, because the variation points offered by the primitives are not offered by the workflow patterns. To support a similar approach as ours, it would be necessary to mine higher-level patterns in workflows that provide guidance on how to assemble the workflow patterns to larger structures.

Semantic Web Services approaches partially support model-based validation because a certain conformance check against the ontology can be performed. In tools like Adonis/Aris, the model type conformance can be checked implicitly through the tool. In general, for MDSD/software factories it is possible to check conformance to the metamodels and constraints. Many MDSD tools support model validation and constraint checking. The UML profiles do not themselves support model validation or constraint checking, but as they are standardized, existing tools can be used.

The more formal approaches in the related work often aim for model-based verification. Our approach is not designed for this goal. Of course, it is possible to define verifiable models through meta-models and extend our approach, but this has not yet been the focus of our work. The same can be assessed for the MDSD/software factories approach.

The evaluation of our approach is summarized in Table 2. As can be seen, none of the related work takes such an integrative view as our approach. Therefore, modeling all aspects of process-driven SOAs was not well supported. The related work rather concentrates on some specific aspects of process-driven SOA, such as flow abstractions, modeling business processes, integration of ontologies and services, verification, etc. Hence, these approaches cannot provide a complete foundation for a model integration approach for process-driven SOA models.

Another distinguishing characteristic of our approach is that it is based on proven practices. In our experience, this helps especially to find the integration spots between the different models and to find adequate modeling abstractions in the various modeling domains.

\section{Conclusion}

In this paper, we have introduced a concept for model-driven development of process-driven SOAs that is based on proven practices. We have especially focused on the aspect of model integration by introducing an approach that is based on a common meta-meta-model from which concrete meta-models for DSLs are derived. In the different DSLs and their respective meta-models, patterns are formalized as pattern primitives, so that the pattern's constraints can be ensured for all instances of all different meta-models. We have shown in the examples how to integrate message flow models, business process models, and architectural models. The approach is, however, applicable for all other kinds of process-driven SOA models for which a formal meta-model can be given. Our tools and DSLs can be flexibly used in model-driven development for formally specifying process-driven SOAs, validating the models, and code generation in executable languages.

\section{References}

[1] BOC Europe. Adonis. http://www.boc-eu.com/, 2006. 
[2] W. Aalst, A. Hofstede, B. Kiepuszewski, and A. Barros. Workflow patterns. Distributed and Parallel Databases, 14:5-51, 2003.

[3] C. Alexander. The Timeless Way of Building. Oxford Univ. Press, 1979.

[4] J. Cardoso and A. Sheth. Semantic e-workflow composition. J. Intell. Inf. Syst., 21(3):191-225, 2003.

[5] M. Champion. Towards a reference architecture for Web services. http://www.idealliance.org/papers/dx_xml03/ papers/04-01-01/04-01-01.pdf, 2004.

[6] DAML Services. OWL-S 1.1 Release. http://www.daml.org/services/owl-s/1.1/, 2004.

[7] N. Desai, A. U. Mallya, A. K. Chopra, and M. P. Singh. Interaction protocols as design abstractions for business processes. IEEE Transactions on Software Engineering, 31(12):1015-1027, 2005.

[8] A. H. Eden and Y. Hirshfeld. LePUS - symbolic logic modeling of object oriented architectures: A case study. In Second Nordic Workshop on Software Architecture - NOSA'99, Ronneby, Sweden, April 1999.

[9] E. Gamma, R. Helm, R. Johnson, and J. Vlissides. Design Patterns: Elements of Reusable Object-Oriented Software. Addison-Wesley, 1994.

[10] T. Gardner. Uml modeling of automated business processes with a mapping to bpel4ws. In ECOOP Workshop on Object Orientation and Web Services, Darmstadt, Germany, July 2003.

[11] C. E. Gerede, R. Hull, O. Ibarra, and J. Su. Automated composition of e-services: Lookaheads. In Proceedings of the International Conference on Service Oriented Computing (ICSOC 2004), pages 252-262, New York, NY, US, June 2004.

[12] J. Greenfield and K. Short. Software Factories: Assembling Applications with Patterns, Frameworks, Models \& Tools. J. Wiley and Sons Ltd., 2004.

[13] R. Heckel, M. Lohmann, and S. Thoene. Towards a uml profile for service-oriented architectures. In Workshop on Model Driven Architecture: Foundations and Applications (MDAFA) 2003, CTIT Technical Report TR-CTIT03-27, University of Twente, Enschede, The Netherlands, June 2003.

[14] C. Hentrich and U. Zdun. Patterns for process-oriented integration in service-oriented architectures. In Proceedings of 11th European Conference on Pattern Languages of Programs (EuroPlop 2006), Irsee, Germany, July 2006.

[15] G. Hohpe and B. Woolf. Enterprise Integration Patterns. Addison-Wesley, 2003.

[16] IDS Scheer. Aris Platform. http://www.idsscheer.de/germany/products/53956, 2006. 
[17] G. Keller, M. Nuettgens, and A.-W. Scheer. Prozessmodellierung auf der grundlage ereignisgesteuerter prozessketten (epk). Technical Report Veroeffentlichungen des Instituts fr Wirtschaftsinformatik (IWi), Heft 89, Universitaet des Saarlandes, 1992.

[18] E. Kindler. On the semantics of epcs: Resolving the vicious circle. Data \& Knowledge Engineering, 56(1):2340, 2006. Elsevier.

[19] J. Mak, C. Choy, and D. Lun. Precise modeling of design patterns in UML. In Proceedings of the 26th International Conference on Software Engineering (ICSE'04), pages 252-261, Edinburgh, Scotland, United Kingdom, 2004. IEEE Computer Society.

[20] T. Mikkonen. Formalizing design patterns. In Proceedings of the 20th International Conference on Software Engineering, pages 115-124, Kyoto, Japan, 1998. IEEE Computer Society.

[21] Object Management Group. Business Process Modeling Notation (BPMN). http://www.bpmn.org/, 2006.

[22] Open Architecture Ware. openArchitectureWare 4.1. http://www.openarchitectureware.org/, 2006.

[23] D. Schmidt and F. Buschmann. Patterns, frameworks, and middleware: Their synergistic relationships. In 25th International Conference on Software Engineering, pages 694-704, May 2003.

[24] M. Solanki, A. Cau, and H. Zedan. Augmenting semantic web service descriptions with compositional specification. In $W W W$ '04: Proceedings of the 13th international conference on World Wide Web, pages 544-552, 2004.

[25] N. Soundarajan and J. O. Hallstrom. Responsibilities and rewards: Specifying design patterns. In Proceedings of the 26th International Conference on Software Engineering, pages 666-675. IEEE Computer Society, 2004.

[26] T. Stahl and M. Voelter. Model-Driven Software Development. J. Wiley and Sons Ltd., 2006.

[27] Sun. Sun reference architectures. http://www.sun.com/service/refarch/, 2004.

[28] The Middleware Company. SOA blueprints. http://www.middlewareresearch.com/soa-blueprints/, 2004.

[29] S. Vinoski. Integration with Web services. IEEE Internet Computing, November/December 2003.

[30] M. Vokac and J. M. Glattetre. Using a domain-specific language and custom tools to model a multi-tier serviceoriented application - experiences and challenges. In Proc. of Model Driven Engineering Languages and Systems, 8th International Conference, MoDELS 2005, pages 492-506, Montego Bay, Jamaica, October 2005.

[31] H. Wada, J. Suzuki, and K. Oba. Modeling non-functional aspects in service oriented architecture. In Proc. of the 2006 IEEE International Conference on Service Computing, Chicago, IL, September 2006.

[32] U. Zdun. Frag. http://frag.sourceforge.net/, 2005. 
[33] U. Zdun. Tailorable language for behavioral composition and configuration of software components. Computer Languages, Systems and Structures: An International Journal, 32(1):56-82, 2006.

[34] U. Zdun and P. Avgeriou. Modeling architectural patterns using architectural primitives. In Proceedings of the 20th ACM Conference on Object-Oriented Programming, Systems, Languages \& Applications (OOPSLA 2005), pages 133-146, San Diego, CA, USA, October 2005. ACM Press.

[35] U. Zdun, C. Hentrich, and W. van der Aalst. A survey of patterns for service-oriented architectures. International Journal of Internet Protocol Technology, 1(3):132-143, 2006.

[36] O. Zimmermann, P. Krogdahl, and C. Gee. Elements of Service-Oriented Analysis and Design: An interdisciplinary modeling approach for SOA projects. http://www-128.ibm.com/developerworks/webservices/library/ ws-soad1/, Jun 2004. 